Comment. Math. Helv. 72 (1997) 216-243

(C) 1997 Birkhäuser Verlag, Basel

$0010-2571 / 97 / 020216-28 \$ 1.50+0.20 / 0$

Commentarii Mathematici Helvetici

\title{
Topological quantum field theory with corners based on the Kauffman bracket
}

Răzvan Gelca

Abstract. We describe the construction of a topological quantum field theory with corners based
on the Kauffman bracket, that underlies the smooth theory of Lickorish, Blanchet, Habegger,
Masbaum and Vogel. We also exhibit some properties of invariants of 3-manifolds with boundary.

Mathematics Subject Classification (1991). 57M25, 57N10.

Keywords. Topological quantum field theory, Kauffman bracket.

\section{Introduction}

The discovery of the Jones polynomial $[\mathrm{J}]$ and its placement in the context of quantum field theory done by Witten [Wi] led to various constructions of topological quantum field theories (shortly TQFT's). A first example of a rigorous theory which fits Witten's framework has been produced by Reshetikhin and Turaev in [RT], and makes use of the representation theory of quantum deformations of $s l(2, C)$ at roots of unity (see also $[\mathrm{KM}])$. Then, an alternative construction based on geometric techniques has been worked out by Kohno in [Ko]. A combinatorial approach based on skein spaces associated to the Kauffman bracket [K1] has been exhibited by Lickorish in [L1] and [L2] and by Blanchet, Habegger, Masbaum, and Vogel in [BHMV1]. All these theories are smooth, in the sense that they define invariants satisfying the Atiyah-Segal set of axioms [A], thus there is a rule telling how the invariants behave under gluing of 3-manifolds along closed surfaces.

In an attempt to give a more axiomatic approach to such a theory, K. Walker described in [Wa] a system of axioms for a TQFT in which one allows gluings along surfaces with boundary, a so called TQFT with corners. This system of axioms includes the Atiyah-Segal axioms, but works for a more restrictive class of TQFT's. From Walker's point of view a TQFT with corners consists out of a minimal amount of information (basic data), and a set of rules for transformations of invariants, which enables us to compute the invariants of all manifolds out of those for some very simple ones. The theory is based on the decompositions of surfaces 
into disks, annuli and pairs of pants, and along with the mapping class group of a surface one considers the groupoid of transformations of the decompositions.

This method is useful in explaining the Reshetikhin-Turaev formula for the invariants of manifolds with or without boundary, in explaining the normalization of these invariants, and in simplifying computations in certain particular cases. Moreover, by identifying the invariant of a colored link in a manifold with the corresponding coordinate of the invariant of the complement of a regular neighborhood of that link, the TQFT with corners also covers Witten's ideas about cutting Wilson lines into pieces (see [Wi]).

We remind the existence of a TQFT with corners for the Turaev-Viro invariant. In that theory one is allowed to cut up to the level of simplices in a triangulation, however the invariants it produces are less fine than the Reshetikhin-Turaev invariants. The approach from this paper allows us to cut down to the level of a handle decomposition, but in return it produces the much finer Reshetikhin-Turaev invariants.

Following partial work from [Wa], in [FK] and [G1] a TQFT with corners associated to the Reshitikhin-Turaev theory was exhibited. We mention that in this construction one encounters a sign obstruction at the level of the groupoid of transformations of decompositions. Its presence is due to the fact that [G2] that the theory is based on the Jones polynomial, whose skein relation is defined for oriented links, so in this case one cannot locally cut and change the orientation of the link. This sign anomaly has been eliminated in [FK] by making use only of half of the irreducible representations, and in [G1] by introducing additional structure on the boundary of manifolds.

In the present paper we construct a TQFT with corners that underlies the smooth TQFT of Lickorish, Blanchet, Habegger, Masbaum and Vogel. It is based on the skein theory of the Kauffman bracket. We make our construction by specifying the basic data and checking that it gives rise to a well defined theory. We make the remark that many elements of the basic data have appeared previously in the literature, however, as far as we know, none of their treatment was done in this context. The paper has many similarities with $[\mathrm{FK}]$, but from many points of view it is much simpler. For example we no longer have to delete half of the labels, we no longer need a special direction in the plane, the analogues of the Clebsch-Gordan are easier to construct and understand.

We point out that there exist several smooth TQFT that correspond to the same invariants for closed manifolds, for example the Reshetikhin-Turaev invariants for closed manifolds appear in both the theories from [RT] and [L], [BHMV1]. However, in [BHMV2] was shown that there is always a canonical TQFT. In our case this is the one of Lickorish, Blanchet, Habegger, Masbaum and Vogel. Consequently, the theory with corners that we construct below is the one canonically associated to the Reshetikhin-Turaev invariants.

In Section 2 we review the definitions from [Wa]. Section 3 starts with a review of facts about skein spaces and then proceeds with the description of the basic data. 
In Section 4 we prove that the basic data gives rise to a well defined TQFT. As a main device involved in the proof we exhibit a tensor contraction formula. In the last section we generalize to surfaces with boundary a well known formula for the invariant of the product of a closed surface with a circle. Next we show that the invariants of 3-manifolds with boundary have a distinguished vector component which satisfies the Kauffman bracket skein relation. As a consequence we deduce from axioms the formula for the invariant of a regular neighborhood of a link, and consequently we give a proof of the Reshetikhin-Turaev formula for closed manifolds. Note that the proof of the formula for manifolds with boundary will appear in a future paper.

\section{Facts about TQFT's with corners}

A TQFT with corners is one that allows gluings of 3-manifolds along surfaces in their boundary that themselves have boundary. In order to be able to understand such a theory we must first briefly describe its objects, the extended surfaces and 3 -manifolds. For an extensive discussion we recommend [Wa]. The adjective "extended" comes from the way the projective ambiguity of the invariants is resolved, which is done, as usually, via an extension of the mapping class group. All surfaces and 3-manifolds throughout the paper are supposed to be piecewise linear, compact and orientable.

In order to fulfill the needs of a TQFT with corners, the concept of extended surface will involve slightly more structure than the usual Lagrangian space, namely the decomposition into disks, annuli and pairs of pants (shortly DAPdecomposition).

Definition. A DAP-decomposition of a surface $\Sigma$ consists of

- a collection of disjoint simple closed curves in the interior of $\Sigma$ that cut $\Sigma$ into elementary surfaces: disks, annuli, and pairs of pants, and an ordering of these elementary surfaces;

- for each elementary surface $\Sigma_{0}$ a numbering of its boundary components, by 1 if $\Sigma_{0}$ is a disk, 1 and 2 if $\Sigma_{0}$ is an annulus, and 1,2 and 3 if $\Sigma_{0}$ is a pair of pants, a parametrization of each boundary component $C$ of $\Sigma_{0}$ by $S^{1}=\{z|| z \mid=1\}$ (the parametrization being compatible with the orientation of $\Sigma_{0}$ under the convention "first out") such that the parametrizations coming from two neighboring elementary surfaces are one the complex conjugate of the other, and fixed disjoint embedded arcs in $\Sigma_{0}$ joining $e^{i \epsilon}$ (where $\epsilon>0$ is small) on the $j$-th boundary component to $e^{-i \epsilon}$ on the $j+1$-st (modulo the number of boundary components of $\Sigma_{0}$ ) (these arcs will be called seams).

An example of a DAP-decomposition is shown in Fig 2.1. Two decompositions are considered identical if they coincide up to isotopy. We also make the conven- 
tion that whenever we talk about the decomposition curves we also include the boundary components of the surface as well.

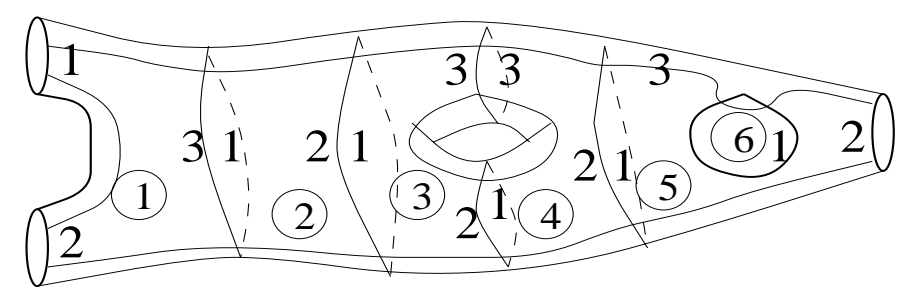

Figure 2.1.

Definition. An extended surface (abbreviated e-surface) is a pair $(\Sigma, D)$ where $\Sigma$ is a surface and $D$ is a DAP-decomposition of $\Sigma$.

Let us note that in the case of smooth TQFT's one is only interested in the Lagrangian subspace spanned by the decomposition curves of $D$ in $H_{1}(\Sigma)$. In our case, we will be interested in the decomposition itself, since we can always arrange the gluing to be along a collection of elementary subsurfaces in the boundary of the 3-manifold. We emphasize that the DAP-decomposition plays the same role as the basis plays for a vector space.

If we change the orientation of a surface, the DAP-decomposition should be changed by reversing all orientations and subsequently by permuting the numbers 2 and 3 in the pairs of pants.

In what follows, we will call a move any transformation of one DAP-decomposition into another. By using Cerf theory [C] one can show that any move can be written as a composition of the elementary moves described in Fig. 2.2 and their inverses, together with the permutation map $P$ that changes the order of elementary surfaces. In the sequel $T_{1}$ will be called a twist, $R$ rotation, the maps $A$ and $D$ contractions of annuli, respectively disks, and their inverses expansions of annuli and disks.

Definition. An extended morphism (shortly e-morphism) is a map between two e-surfaces $(f, n):\left(\Sigma_{1}, D_{1}\right) \rightarrow\left(\Sigma_{2}, D_{2}\right)$ where $f$ is a homeomorphism and $n$ is an integer.

Note that such an e-morphism can be written as a composition of a homeomorphism $(f, 0):\left(\left(\Sigma_{1}, D_{1}\right) \rightarrow\left(\Sigma_{2}, f\left(D_{1}\right)\right)\right.$, a move $\left(\Sigma_{2}, f\left(D_{1}\right)\right) \rightarrow\left(\Sigma_{2}, D_{2}\right)$ and the morphism $(0, n):\left(\Sigma_{2}, D_{2}\right) \rightarrow\left(\Sigma_{2}, D_{2}\right)$. Note also that the moves from Fig 2.2 have the associated homeomorphism equal to the identity.

The set of e-morphisms is given a groupoid structure by means of the following composition law. For $\left(f_{1}, n_{1}\right):\left(\Sigma_{1}, D_{1}\right) \rightarrow\left(\Sigma_{2}, D_{2}\right)$ and $\left(f_{2}, n_{2}\right):\left(\Sigma_{2}, D_{2}\right) \rightarrow$ $\left(\Sigma_{3}, D_{3}\right)$ let 


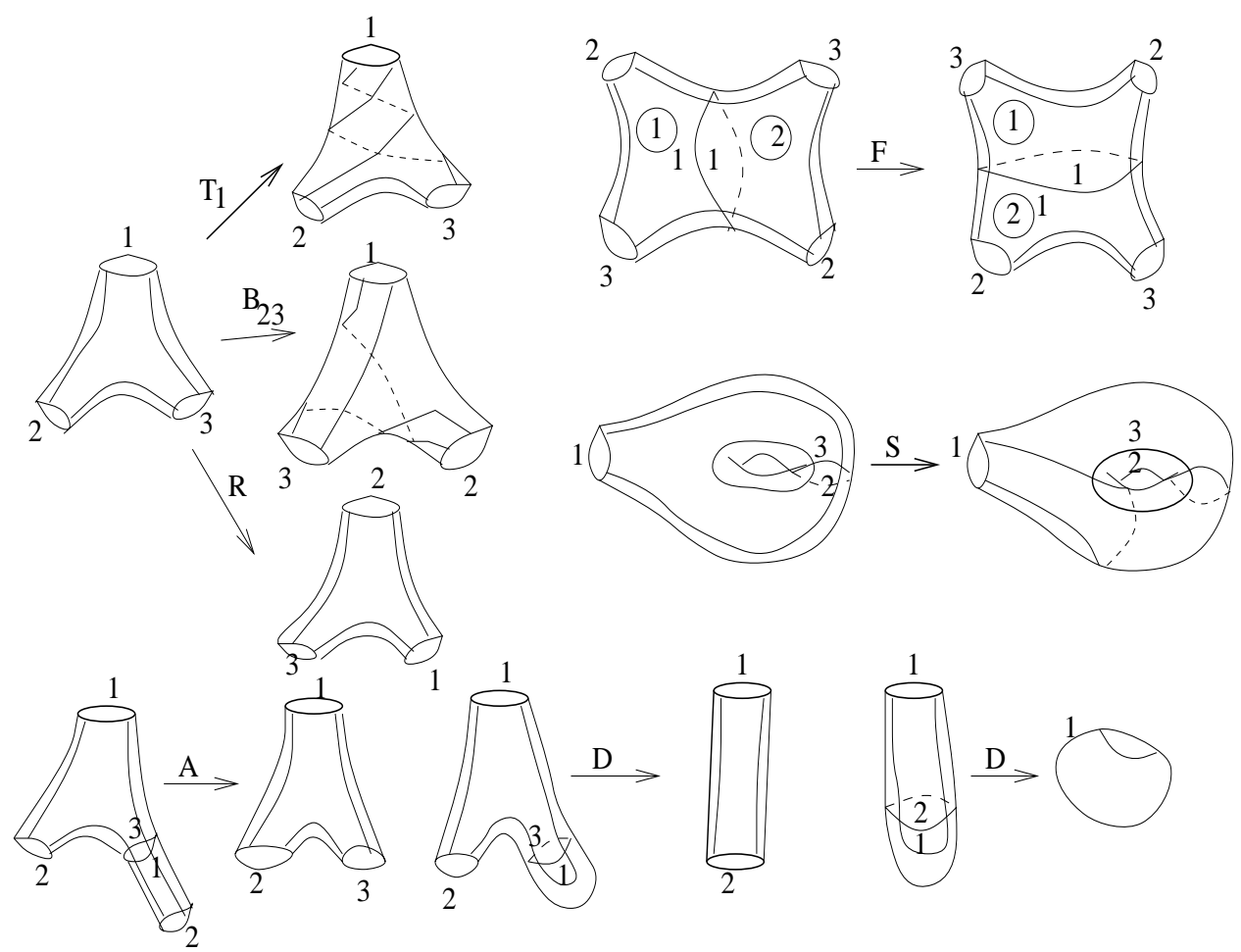

Figure 2.2.

$$
\left(f_{2}, n_{2}\right)\left(f_{1}, n_{1}\right):=\left(f_{2} f_{1}, n_{1}+n_{2}-\sigma\left(\left(f_{2} f_{1}\right)_{*} L_{1},\left(f_{2}\right)_{*} L_{2}, L_{3}\right)\right.
$$

where $\sigma$ is Wall's nonadditivity function [W] and $L_{i} \subset H_{1}\left(\Sigma_{i}\right)$ is the subspace generated by the decomposition curves of $D_{i}, i=1,2,3$.

Let us now review some facts about extended 3-manifolds.

Definition. The triple $(M, D, n)$ is called an extended 3-manifold (e-3-manifold) if $M$ is a 3 -manifold, $D$ is a DAP-decomposition of $\partial M$ and $n \in \mathbf{Z}$.

The boundary operator, disjoint union and mapping cylinder are defined in the canonical way, namely $\partial(M, D, n)=(\partial M, D),\left(M_{1}, D_{1}, n_{1}\right) \sqcup\left(M_{2}, D_{2}, n_{2}\right)=\left(M_{1} \sqcup\right.$ $\left.M_{2}, D_{1} \sqcup D_{2}, n_{1}+n_{2}\right)$ and for $(f, n):\left(\Sigma_{1}, D_{1}\right) \rightarrow\left(\Sigma_{2}, D_{2}\right), I_{(f, n)}=\left(I_{f}, D, n\right)$, with the only modification that in $I_{f}$ we identify the boundary components of $-\Sigma_{1}$ with those of $\Sigma_{2}$ that they get mapped onto, thus $\partial I_{f}=-\Sigma_{1} \cup \Sigma_{2}$ and $D=D_{1} \cup D_{2}$. More complicated is the gluing of e-3-manifolds, which is done as follows.

Definition. Let $(M, D, n)$ be an e-3-manifold and $\left(\Sigma_{1}, D_{1}\right)$ and $\left(\Sigma_{2}, D_{2}\right)$ be two 
disjoint surfaces in its boundary. Let $(f, m):\left(\Sigma_{1}, D_{1}\right) \rightarrow\left(\Sigma_{2}, D_{2}\right)$ be an emorphism. Define the gluing of $(M, D, n)$ by $(f, m)$ to be

$$
(M, D, n)_{(f, m)}:=\left(M_{f}, D^{\prime}, m+n-\sigma\left(K, L_{1} \oplus L_{2}, \Delta^{-}\right)\right)
$$

where $M_{f}$ is the gluing of $M$ by $f, D^{\prime}$ is the image of $D$ under this gluing, $\sigma$ is Wall's nonadditivity function, $K$ is the kernel of $H_{1}\left(\Sigma_{1} \cup \Sigma_{2}\right) \rightarrow H_{1}(M) / J$, $J$ being the subspace of $H_{1}(\partial M)$ spanned by the decomposition curves lying in the complement of $\operatorname{int}\left(\Sigma_{1} \cup \Sigma_{2}\right), L_{i}$ are the subspaces of $H_{1}\left(\Sigma_{i}\right)$ generated by the decomposition curves of $D_{i}$ and $\Delta^{-}=\left\{\left(x,-f_{*}(x)\right), x \in H_{1}\left(\Sigma_{1}\right)\right\}$.

For a geometric explanation of this definition see [Wa].

In order to define a TQFT we also need a finite set of labels $\mathcal{L}$, with a distinguished element $0 \in \mathcal{L}$. Consider the category of labeled extended surfaces (le-surfaces) whose objects are e-surfaces with the boundary components labeled by elements in $\mathcal{L}$ (le-surfaces), and whose morphisms are the e-morphisms that preserve labeling (called labeled extended morphism and abbreviated le-morphisms). An le-surface is thus a triple $(\Sigma, D, l)$, where $l$ is a labeling function.

Following $[\mathrm{Wa}]$ we define a TQFT with label set $\mathcal{L}$ to consist out of

- a functor $V$ from the category of le-surfaces to that of finite dimensional vector spaces, called modular functor,

- a partition function $Z$ that associates to each 3-manifold a vector in the vector space of its boundary.

The two should satisfy the following axioms:

(2.1) (disjoint union) $V\left(\Sigma_{1} \sqcup \Sigma_{2}, D_{1} \sqcup D_{2}, l_{1} \sqcup l_{2}\right)=V\left(\Sigma_{1}, D_{1}, l_{1}\right) \otimes V\left(\Sigma_{2}, D_{2}, l_{2}\right)$;

(2.2) (gluing for $V$ ) Let $(\Sigma, D)$ be an le-surface, $C, C^{\prime}$ two subsets of boundary components of $(\Sigma)$, and $g: C \rightarrow C^{\prime}$ the homeomorphism which is the parametrization reflecting map. Let $\Sigma_{g}$ be the gluing of $\Sigma$ by $g$, and $D_{g}$ the DAP-decomposition induced by $D$. Then, for a certain labeling $l$ of $\partial \Sigma$ we have

$$
V\left(\Sigma_{g}, D_{g}, l\right)=\bigoplus_{x \in \mathcal{L}(C)} V(\Sigma, D,(l, x, x))
$$

where the sum is over all labelings of $C$ and $C^{\prime}$ by $x$.

(2.3) (duality) $V(\Sigma, D, l)^{*}=V(-\Sigma,-D, l)$ and the identifications $V(\Sigma, D, l)=$ $V(-\Sigma,-D, l)^{*}$ and $V(-\Sigma,-D, l)=V(\Sigma, D, l)^{*}$ are mutually adjoint. Moreover, the following conditions should be satisfied

- if $(f, n)$ is an le-morphism between to le-surfaces, then $V(\bar{f},-n)$ is the adjoint inverse of $V(f, n)$, where we denote by $\bar{f}$ the homeomorphism induced between the surfaces with reversed orientation.

- if $\alpha_{1} \otimes \alpha_{2} \in V\left(\Sigma_{1}, D_{1}, l_{1}\right) \otimes V\left(\Sigma_{2}, D_{2}, l_{2}\right)$ and $\beta_{1} \otimes \beta_{2} \in V\left(-\Sigma_{1},-D_{1}, l_{1}\right)$ $\otimes V\left(-\Sigma_{2},-D_{2}, l_{2}\right)$ then $<\alpha_{1} \otimes \alpha_{2}, \beta_{1} \otimes \beta_{2}>=<\alpha_{1}, \beta_{1}><\alpha_{2}, \beta_{2}>$,

- there exists a function $S: \mathcal{L} \rightarrow \mathbf{C}^{*}$ such that with the notations from axiom $(2.2)$ if $\oplus_{x} \alpha_{x} \in \bigoplus_{x \in \mathcal{L}(C)} V(\Sigma, D,(l, x, x))$ and $\oplus_{x} \beta_{x} \in \bigoplus_{x \in \mathcal{L}(C)} V(-\Sigma,-D,(l, x, x))$ then the pairing on the glued surface is given by $<\oplus_{x} \alpha_{x}, \oplus_{x} \beta_{x}>=\sum_{x} S(x)<$ $\alpha_{x}, \beta_{x}>$, where $x=\left(x_{1}, x_{2}, \cdots, x_{n}\right)$ and $S(x)=S\left(x_{1}\right) S\left(x_{2}\right) \cdots S\left(x_{n}\right)$;

(2.4) (empty surface) $V(\emptyset)=\mathbf{C}$;

(2.5) (disk) If $\mathbf{D}$ is a disk $V(\mathbf{D}, m)=\mathbf{C}$ if $m=0$ and 0 otherwise; 
(2.6) (annulus) If $A$ is an annulus then $V(A,(m, n))=\mathbf{C}$ if $m=n$ and 0 otherwise;

(2.7) (disjoint union for $Z) Z\left(\left(M_{1}, D_{1}, n_{1}\right) \sqcup\left(M_{2}, D_{2}, n_{2}\right)\right)=Z\left(M_{1}, D_{1}, n_{1}\right) \otimes$ $Z\left(M_{2}, D_{2}, n_{2}\right)$

(2.8) (naturality) Let $(f, 0):\left(M_{1}, D, n\right) \rightarrow\left(M_{2}, f(D), n\right)$. Then $V\left(f \mid \partial\left(M_{1}, D, n\right)\right) Z\left(M_{1}, D, n\right)=Z\left(M_{2}, f(D), n\right)$.

(2.9) (gluing for $Z$ ) Let $\left(\Sigma_{1}, D_{1}\right),\left(\Sigma_{2}, D_{2}\right) \subset \partial(M, D, m)$ be disjoint, and let $(f, n):\left(\Sigma_{1}, D_{1}\right) \rightarrow\left(\Sigma_{2}, D_{2}\right)$. Then by $(2.2)$

$V(\partial(M, D, m))=\bigoplus_{l_{1}, l_{2}} V\left(\Sigma_{1}, D_{1}, l_{1}\right) \otimes V\left(\Sigma_{2}, D_{2}, l_{2}\right) \otimes V\left(\partial(M, D, m) \backslash\left(\left(\Sigma_{1}, D_{1}\right) \cup\right.\right.$ $\left.\left(\Sigma_{2}, D_{2}\right), l_{1} \cup l_{2}\right)$

hence $Z(M, D, m)=\bigoplus_{l_{1}, l_{2}} \sum_{j} \alpha_{l_{1}}^{(j)} \otimes \beta_{l_{2}}^{(j)} \otimes \gamma_{l_{1}, l_{2}}^{(j)}$. The axiom states that

$$
Z\left((M, D, m)_{(f, n)}\right)=\oplus_{l} \sum_{j}<V(f, n) \alpha_{l}^{(j)}, \beta_{l}^{(j)}>\gamma_{l l}^{(j)}
$$

- where $l$ runs through all labelings of $\partial \Sigma_{1}$;

(2.10) (mapping cylinder axiom) For $(i d, 0):(\Sigma, D) \rightarrow(\Sigma, D)$ we have

$$
Z\left(I_{(i d, 0)}\right)=\oplus_{l \in \mathcal{L}(\partial \Sigma)} i d_{l}
$$

where $i d_{l}$ is the identity matrix in $V(\Sigma, D, l) \otimes V(\Sigma, D, l)^{*}=V(\Sigma, D, l)$

$\otimes V(-\Sigma,-D, l)$.

\section{The basic data}

In order to construct a TQFT with corners one needs to specify a certain amount of information, called basic data, from which the modular functor and partition function can be recovered via the axioms. Note that the partition function is completely determined by the modular functor, so we only need to know that latter. Moreover, the modular functor is determined by the vector spaces associated to le-disks, annuli and pairs of pants, and by the linear maps associated to lemorphisms. An important observation is that the matrix of a morphism $V(f, 0)$, where $(f, 0):\left(\Sigma_{1}, D\right) \rightarrow\left(\Sigma_{2}, f(D)\right)$, is the identity matrix, so one only needs to know the values of the functor for moves, hence for the elementary moves described in Fig. 2.2. Of course we also need to know its value for the map $C=(i d, 1)$.

The possibility of relating our theory to the Kauffman bracket depends on the choice of basic data. Our construction is inspired by [L1]. We will review the notions we need from that paper and then proceed with our definitions.

Let $\Sigma$ be a surface with a collection of $2 n$ points on its boundary $(n \geq 0)$. A link diagram in $\Sigma$ is an immersed compact 1-manifold $L$ in $\Sigma$ with the property that $L \cap \partial \Sigma=\partial L, \partial L$ consists of the $2 n$ distinguished point on $\partial \Sigma$, the singular points of $L$ are in the interior of $\Sigma$ and are transverse double points, and for each such point the "under" and "over" information is recorded.

Let $A \in \mathbf{C}$ be fixed. The skein vector space of $\Sigma$, denoted by $\mathcal{S}(\Sigma)$, is defined to be the complex vector space spanned by all link diagrams factored by the following two relations:

a). $L \cup($ trivial closed curve $)=-\left(A^{2}+A^{-2}\right) L$, 
b). $L_{1}=A L_{2}+A^{-1} L_{3}$,

where $L_{1}, L_{2}$ and $L_{3}$ are any three diagrams that coincide except in a small disk, where they look like in Fig. 3.1.

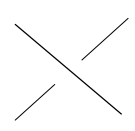

$\mathrm{L}_{1}$

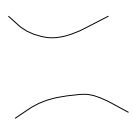

$\mathrm{L}_{2}$

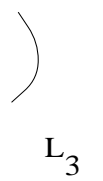

Figure 3.1.

For simplicity, from now on, whenever in a diagram we have an integer, say $k$, written next to a strand we will actually mean that we have $k$ parallel strands there. Also rectangles (coupons) inserted in diagrams will stand for elements of the skein space of the rectangle inserted there.

Three examples are useful to consider. The first one is the skein space of the plane, which is the same as the one of the sphere, and it is well known that it is isomorphic to $\mathbf{C}$.

The second example is that of an annulus $A$ with no points on the boundary. It is also a well known fact that $\mathcal{S}(A)$ is isomorphic to the ring of polynomials $\mathbf{C}[\alpha]$, (if endowed with the multiplication defined by the gluing of annuli). The independent variable $\alpha$ is the diagram with one strand parallel to the boundary of the annulus. Recall from [L1] that every link diagram $L$ in the plane determines a map

$$
<\cdot, \cdot, \cdots, \cdot>_{L}: \mathcal{S}(A) \times \mathcal{S}(A) \times \cdots \mathcal{S}(A) \rightarrow \mathcal{S}\left(\mathbf{R}^{2}\right)
$$

obtained by first expanding each component of $L$ to an annulus via the blackboard framing and then homeomorphically mapping $A$ onto it.

The third example is the skein space of a disk with $2 n$ points on the boundary. If the disk is viewed as a rectangle with $n$ points on one side and $n$ on the opposite, then we can define a multiplication rule on the skein space by juxtaposing rectangles, obtaining the Temperley-Lieb algebra $T L_{n}$. Recall that $T L_{n}$ is generated by the elements $1, e_{1}, e_{2}, \cdots, e_{n-1}$, where $e_{i}$ is described in Fig. 3.2.

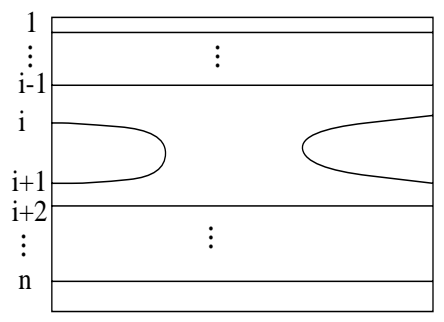

Figure 3.2. 
There exists a map from $T L_{n}$ to $\mathcal{S}\left(\mathbf{R}^{2}\right)$ obtained by closing the elements in $T L_{n}$ by $n$ parallel arcs. This map plays the role of a quantum trace. It splits in a canonical way as $T L_{n} \rightarrow \mathcal{S}(A) \rightarrow \mathcal{S}\left(\mathbf{R}^{2}\right)$ by first closing the elements in an annulus and then including them in a plane.

At this moment we recall the definition of the Jones-Wenzl idempotents [We]. They are of great importance for our construction, since they mimic the behavior of the finite dimensional irreducible representations from the Reshetikhin-Turaev theory [RT]. For this let $r>1$ be an integer (which will be the level of our TQFT). Let $A=e^{i \pi /(2 r)}$. Recall that for each $n$ one denotes by $[n]$ the quantized integer $\left(A^{2 n}-A^{-2 n}\right) /\left(A^{2}-A^{-2}\right)$.

The Jones-Wenzl idempotents are the unique elements $f^{(n)} \in T L_{n}, 0 \leq n \leq$ $r-1$, that satisfy the following properties:

1) $f^{(n)} e_{i}=0=e_{i} f^{(n)}$, for $0 \leq i \leq n-1$,

2) $\left(f^{(n)}-1\right)$ belongs to the algebra generated by $e_{1}, e_{2}, \cdots, e_{n-1}$,

3) $f^{(n)}$ is an idempotent,

4) $\Delta_{n}=(-1)^{n}[n+1]$

where $\Delta_{n}$ is the image of $f^{(n)}$ through the map $T L_{n} \rightarrow \mathcal{S}\left(\mathbf{R}^{2}\right)$.

In the sequel we will have to work with the square root of $\Delta_{n}$ so we make the notation $d_{n}=i^{n} \sqrt{[n+1]}$, thus $\Delta_{n}=d_{n}^{2}$.

Following [L1], in a diagram we will always denote $f^{(n)}$ by an empty coupon (see Fig. 3.3).

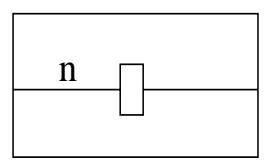

Figure 3.3.

The image of $f^{(n)}$ through the map $T L_{n} \rightarrow \mathcal{S}(A)$ will be denoted by $S_{n}(\alpha)$. We will also need the element $\omega \in \mathcal{S}(A), \omega=\sum_{n=0}^{r-2} d_{n}^{2} S_{n}(\alpha)$. Given a link diagram $L$ in the plane, whenever we label one of its components by $\omega$ we actually mean that we inserted $\omega$ in the way described in the definition of $\langle\cdot, \cdot, \cdots, \cdot\rangle_{L}$. Note that one can perform handle slides (also called second Kirby moves [Ki]), over components labeled by $\omega$ without changing the value of the diagram (see [L1]).

Now we can define the basic data for a TQFT in level $r$, where $r$, as said, is an integer greater than 1 . Let $\mathcal{L}=\{0,1, \cdots, r-2\}$. Make the notation $X=$ $(i \sqrt{2 r}) /\left(A^{2}-A^{-2}\right)$, that is $X^{2}=\sum d_{n}^{4}=<\omega>_{U}$, where $U$ is the unknot with zero framing (see [L1]).

Notice that by gluing two disks along the boundary we get a pairing map $\mathcal{S}(\mathbf{D}, 2 n) \times \mathcal{S}(\mathbf{D}, 2 n) \rightarrow \mathcal{S}\left(S^{2}\right)=\mathbf{C}$, hence we can view $\mathcal{S}(\mathbf{D}, 2 n)$ as a set of functionals acting on the skein space of the exterior. In what follows, whenever we mention the skein space of a disk, we will always mean the skein space as a 
set of functionals in this way. For example this will enable us to get rid of the diagrams that have a strand labeled by $r-1$ (see also [L1], [K2]). The point of view is similar to that of factoring by the bad part of a representation (the one of quantum trace 0) in the Reshetikhin-Turaev setting.

To a disk with boundary labeled by 0 we associate the vector space $V_{0}$ which is the skein space of a disk with no points on the boundary. Of course for any other label $a$ we put $V_{a}=0$. It is obvious that $V_{0}=\mathbf{C}$. We let $\beta_{0}$ be the empty diagram.

a)

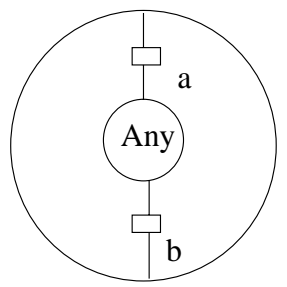

b)

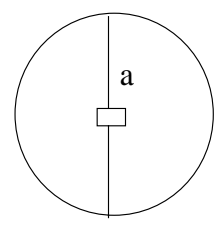

Figure 3.4.

To an annulus with boundary components labeled by $a$ and $b$ we associate the vector space $V_{a b}$ which is the subspace of $\mathcal{S}(\mathbf{D}, a+b)$ spanned by all diagrams of the form indicated in Fig. 3.4. a), where in the smaller disk can be inserted any diagram from $\mathcal{S}(\mathbf{D}, a+b)$. The first condition in the definition of the JonesWenzl idempotents implies that $V_{a b}=0$ if $a \neq b$ and $V_{a a}$ is one dimensional and is spanned by the diagram from Fig. 3.4. b). We will denote by $\beta_{a a}$ this diagram multiplied by $1 / d_{a}$, where we recall that $d_{a}=i^{a} \sqrt{[a+1]}$. The element $\beta_{a a}$ has the property that paired with itself on the outside gives 1 .

To a pair of pants with boundary components labeled by $a, b$, and $c$ we put into correspondence the space $V_{a b c}$, which is the space spanned by all diagrams of the form described in Fig. 3.5. a), where in the inside disk we allow any diagram from $\mathcal{S}(\mathbf{D}, a+b+c)$.

a)

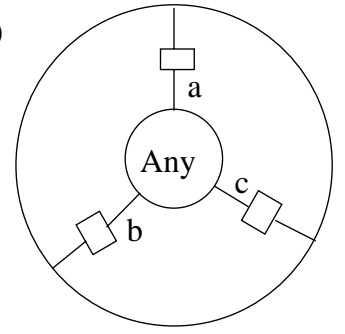

b)

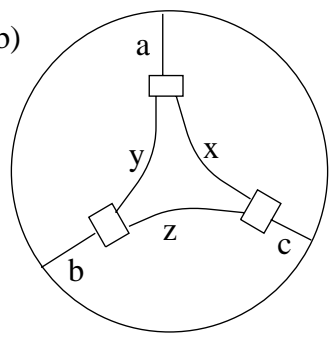

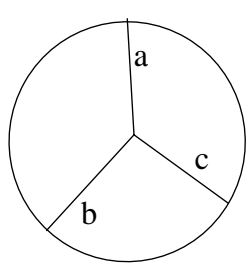

Figure 3.5.

The reader will notice that there is some ambiguity in this definition. To make 
it rigorous, we have to mark a point on the circle, from which all points are counted. We will keep this in mind although we will no longer mention it.

The results from [K2] and [L1] show that $V_{a b c}$ can either be one dimensional or it is equal to zero. The triple $(a, b, c)$ is said to be admissible if $V_{a b c} \neq 0$. This is exactly the case when $a+b+c$ is even, $a+b+c \leq 2(r-2)$ and $a \leq b+c$, $b \leq a+c, c \leq a+b$. In this case the space $V_{a b c}$ is spanned by the triad introduced by Kauffman [K2] which is described in Fig. 3.5. b). Here the numbers $x, y, z$ satisfy $a=x+y, b=y+z, c=z+x$.

(1)

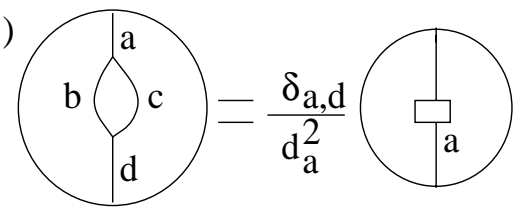

(2)
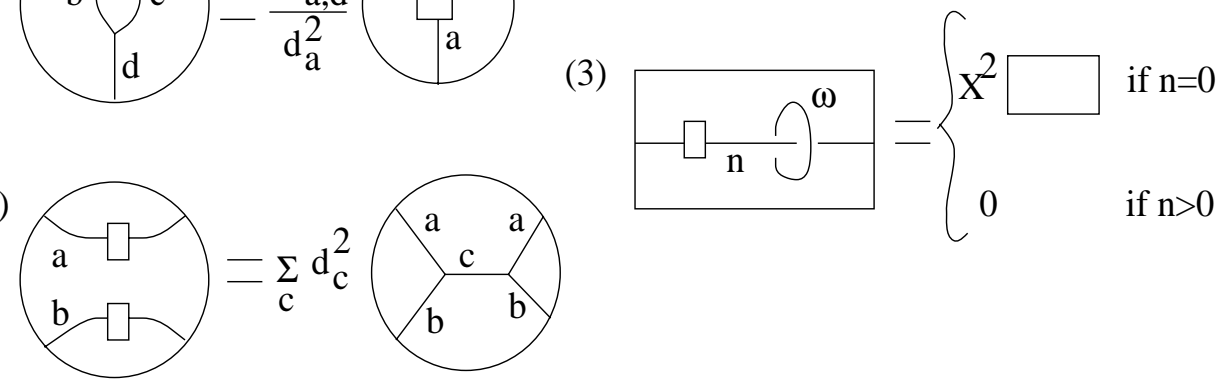

Figure 3.6.

In [L2] it is shown that if we pair the diagram from Fig. 3.5. b) with the one corresponding to $V_{a c b}$ on the outside we get the complex number $\theta(x+y, y+$ $z, z+x)=\left(\Delta_{x+y+z} ! \Delta_{x-1} ! \Delta_{y-1} ! \Delta_{z-1} !\right) /\left(\Delta_{y+z-1} ! \Delta_{z+x-1} ! \Delta_{x+y-1} !\right)$, where $\Delta_{n}=$ $\Delta_{1} \Delta_{2} \cdots \Delta_{n}$ and $\Delta_{-1}=1$. Thus if we denote by $\beta_{a b c}$ the product of this diagram with $\left(d_{x+y+z} ! d_{x-1} ! d_{y-1} ! d_{z-1} !\right)^{-1}\left(d_{y+z-1} ! d_{z+x-1} ! d_{x+y-1} !\right)=1 / \sqrt{\theta(a, b, c)}$ (with the same convention for factorials), then $\beta_{a b c}$ paired on the outside with $\beta_{a c b}$ will give 1.

In diagrams, whenever we have a $\beta_{a b c}$ we make the notation from Fig. 3.5. c). This notation is different from the one with a dot in the middle from [L1], in the sense that we have a different normalization! We prefer this notation because it will simplify diagrams in the future, so whenever in a diagram we have a trivalent vertex, we consider that we have a $\beta$ inserted there. In particular, a diagram that looks like the Greek letter $\theta$ will be equal to 1 in $\mathcal{S}\left(\mathbf{R}^{2}\right)$. The elements $\beta_{a b c}$ are the analogues of the quantum Clebsch-Gordan coefficients.

In the sequel we will need the three identities described in Fig. 3.6, whose proofs can be found in [L1]. Here $\delta_{a d}$ is the Kronecker symbol.

Let us define the dual spaces. It is natural to let the dual of $V_{0}$ to be $V_{0}$, that of $V_{a a}$ to be $V_{a a}$, and that of $V_{a b c}$ to be $V_{a c b}$. However the pairings will look peculiar. This is due to the fact that we want the mapping cylinder axiom to be satisfied. So we let $<,>: V_{0} \times V_{0} \rightarrow \mathbf{C}$ be defined by $<\beta_{0}, \beta_{0}>=1,<,>: V_{a a} \times V_{a a} \rightarrow \mathbf{C}$ 


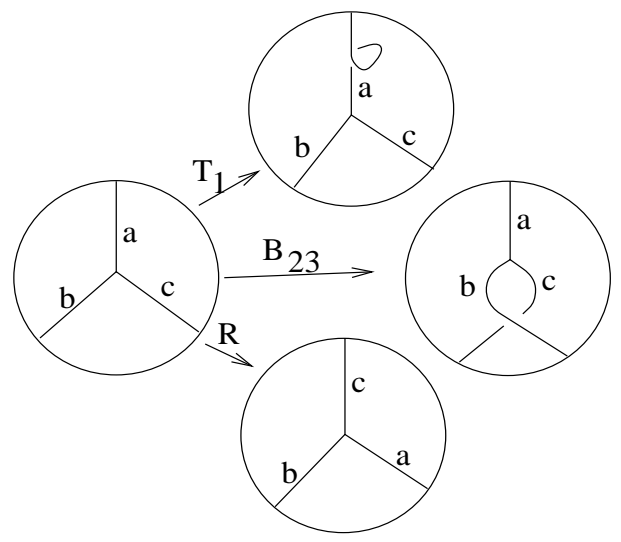

Figure 3.7.

be defined by $<\beta_{a a}, \beta_{a a}>=X / d_{a}^{2}$, and $<,>: V_{a b c} \times V_{a c b} \rightarrow \mathbf{C}$ be defined by $<\beta_{a b c}, \beta_{a c b}>=X^{2} /\left(d_{a} d_{b} d_{c}\right)$.

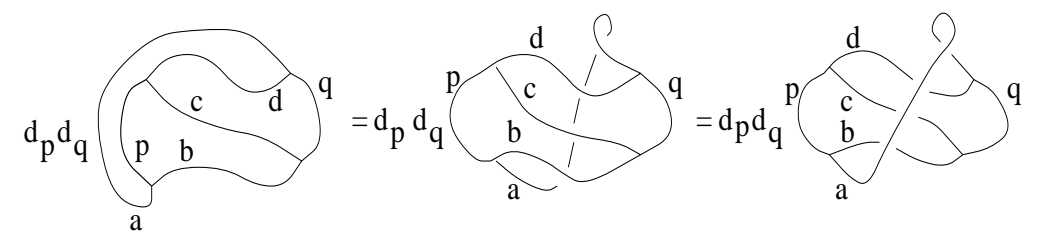

Figure 3.8.

Before we define the morphisms associated to the elementary moves we make the convention that for any e-morphism $f$ we will denote $V(f)$ also by $f$.

The morphisms corresponding to the three elementary moves on a pair of pants are described in Fig. 3.7. Further, we let $F: \bigoplus_{p} V_{p a b} \otimes V_{p c d} \rightarrow \bigoplus_{q} V_{q d a} \otimes V_{q b c}$ be defined by $F \beta_{p a b} \otimes \beta_{p c d}=\sum_{q} f_{a b c d p q} \beta_{q d a} \otimes \beta_{q b c}$ the coefficients $f_{a b c d p q}$ being given by any of the three equal diagrams from Fig. 3.8. Note that $f_{a b c d p q}=d_{p}^{-1} d_{q}\left\{\begin{array}{l}b c p \\ a d q\end{array}\right\}$ where $\left\{\begin{array}{l}b c p \\ a d q\end{array}\right\}$ are the $6 \mathrm{j}$-symbols.

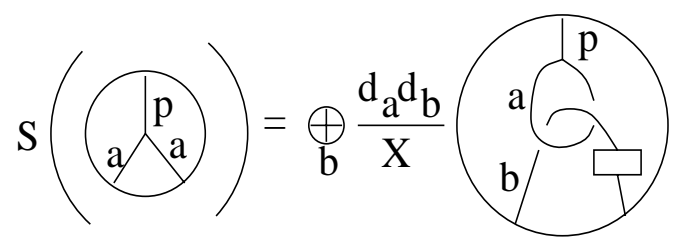

Figure 3.9 
a)

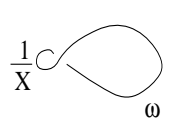

b)

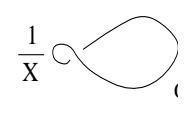

Figure 3.10

Also the map $S: \bigoplus V_{p a a} \rightarrow \bigoplus V_{p b b}$ is described in Fig. 3.9.

The maps $A, D$ and $P$ are given by relations of the form $A\left(x \otimes \beta_{a a}\right)=x$, $D\left(\beta_{a a 0} \otimes \beta_{0}\right)=\beta_{a a}$ and $P(x \otimes y)=y \otimes x$. The map $C$ is the multiplication by the value of the diagram described in Fig. 3.10. a). Note that Lemma 4 in [L1] implies that the inverse of $C$ is the multiplication by the diagram from Fig. 3.11. b). Finally, $S(a)=d_{a}^{2} / X, a \in \mathcal{L}$.

Remark. The reader should note that the crossings from all these diagrams are negative. We make this choice because, returning to the analogy with vector spaces, all the maps we defined behave like changes of basis rather than like morphisms.

\section{The compatibility conditions}

In order for the basic data to give rise to a well defined TQFT, it has to satisfy certain conditions. A list of such conditions has been exhibited in [Wa] (see also $[\mathrm{MS}]$ ), by making use of techniques of Cerf theory similar to those from [HT]. The first group of relations, the so called Moore-Seiberg equations, are the conditions that have to be satisfied in order for the modular functor to exist. They are as follows:

1. at the level of a pair of pants:

a) $T_{1} B_{23}=B_{23} T_{1}, T_{2} B_{23}=B_{23} T_{3}, T_{3} B_{23}=B_{23} T_{2}$, where $T_{2}=R T_{1} R^{-1}$ and $T_{3}=R^{-1} T_{1} R$,

b) $B_{23}^{2}=T_{1} T_{2}^{-1} T_{3}^{-1}$,

c) $R^{3}=1$,

d) $R B_{23} R^{2} B_{23} R B_{23} R^{2}=1$,

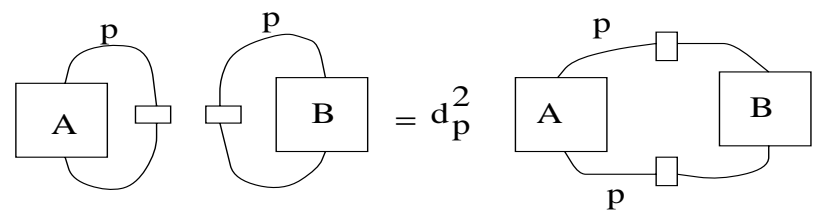

Figure 4.1. 
2. relations defining inverses:
a) $P^{(12)} F^{2}=1$,
b) $T_{3}^{-1} B_{23}^{-1} S^{2}=1$,

3. relations coming from "codimension 2 singularities":

a) $P^{(13)} R^{(2)} F^{(12)} R^{(2)} F^{(23)} R^{(2)} F^{(12)} R^{(2)} F^{(23)} R^{(2)} F^{(12)}=1$,

b) $T_{3}^{(1)} F B_{23}^{(1)} F B_{23}^{(1)} F B_{23}^{(1)}=1$,

c) $C^{-1} B_{23}^{-1} T_{3}^{-2} S T_{3}^{-1} S T_{3}^{-1} S=1$,

d) $R^{(1)}\left(R^{(2)}\right)^{-1} F S^{(1)} F B_{23}^{(2)} B_{23}^{(1)}=F S^{(2)} T_{3}^{(2)}\left(T_{1}^{(2)}\right)^{-1} B_{23}^{(2)} F$,

4. relations involving annuli and disks:

a) $F\left(\beta_{p}^{m n} \otimes \beta_{p}^{p 0}\right)=\beta_{m}^{0 m} \otimes \beta_{m}^{n p}$,

b) $A_{2}^{(12)} D_{3}^{(13)}=D_{2} D_{3}^{(13)}$

c) $A^{(12)} A^{(23)}=A^{(23)} A^{(12)}$,
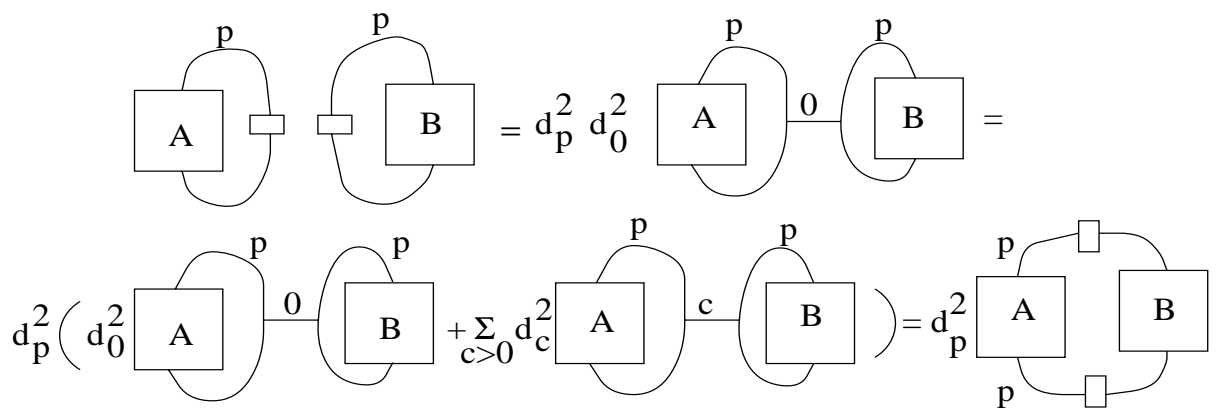

Figure 4.2 .

5. relations coming from duality:

-for any elementary move $f$, one must have $f^{+}=\bar{f}$, where $f^{+}$is the adjoint of $f$ with respect to the pairing, and $\bar{f}$ is the morphism induced by $f$ on the surface with reversed orientation,

6. relations expressing the compatibility between the pairing, and moves $A$ and $D$ :

a) $<\beta_{m}^{m}, \beta_{m}^{m}>=S(m)^{-1}$

b) $<\beta_{m}^{m 0}, \beta_{m}^{m 0}>=S(0)^{-1} S(m)^{-1}$.

In addition one also has to consider two conditions that guarantee that the partition function is well defined.

7. a) $S(m)=S_{0 m}$ where $\left[S_{x y}\right]_{x, y}$ is the matrix of move $S$ on the torus (which can be thought as the punctured torus capped with a disk),

b) $F\left(\beta_{0}^{m m} \otimes \beta_{0}^{n n}\right)=\bigoplus S(m)^{-1} S(n)^{-1} i d_{p m n}$ where $i d_{p m n}$ is the identity matrix in $\left(V_{p m n}\right)^{*} \otimes V_{p m n}$.

In all these relations, the superscripts in parenthesis indicate the index of the elementary surface(s) on which the map acts, and the subscripts indicate the number of the boundary component. 

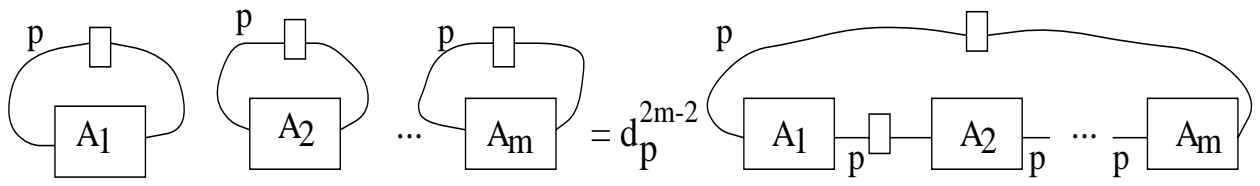

Figure 4.3.

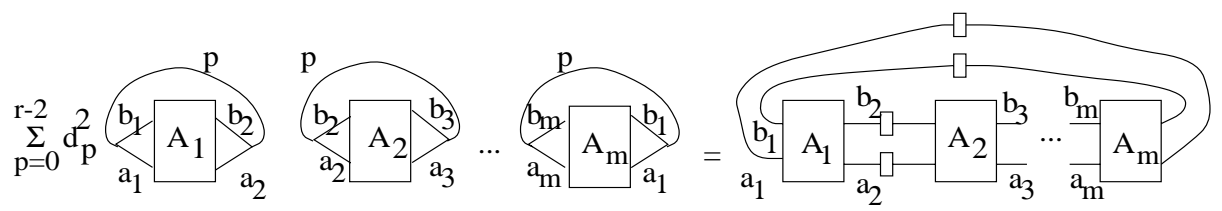

Figure 4.4.

We will prove that our basic data satisfies these relations. For the proof we will need a contraction formula similar to the tensor contraction formula that one encounters in the case of TQFT's based on representations of Hopf algebras (see $[\mathrm{T}],[\mathrm{FK}],[\mathrm{G} 1])$.

Lemma 4.1. For any $A, B \in T L_{p}$ the equality from Fig. 4.1 holds.

Proof. The proof is contained in Fig. 4.2. In this chain of equalities the first one follows from the way we defined the $\beta$ 's, the second one holds because the sum that appears in the third term is zero (by the first property of Jones-Wenzl idempotents, since such an idempotent lies on the strand labeled by $c$; more explanations about this phenomenon can be found in [L1] and $[R])$, and the last equality follows from identity (2) in Fig. 3.6.

Lemma 4.2. If $A_{1}, A_{2}, \cdots, A_{m} \in T L_{p}$ then the identity from Fig. 4.3 holds.

Proof. This result follows by induction from Lemma 4.1.

Theorem 4.1. Suppose that $A_{i} \in \mathcal{S}\left(\mathbf{D}, a_{i}+b_{i}+a_{i+1}+b_{i+1}\right), i=1,2, \cdots, m$, where $a_{i}$ and $b_{i}$ are integers with $a_{m+1}=a_{1}$ and $b_{m+1}=b_{1}$. Then the identity described in Fig. 4.4 holds.

Proof. By Lemma 4.2, the left hand side is equal to the expression described in Fig. 4.5. a).

On the other hand, if $p \neq q$, by using the identity (2) from Fig. 3.6, we get the chain of equalities from Fig. 4.6, where the last one follows from the fact that on the strand labeled by $c$ there is a Jones-Wenzl idempotent and using the first property of these idempotents. 
a)

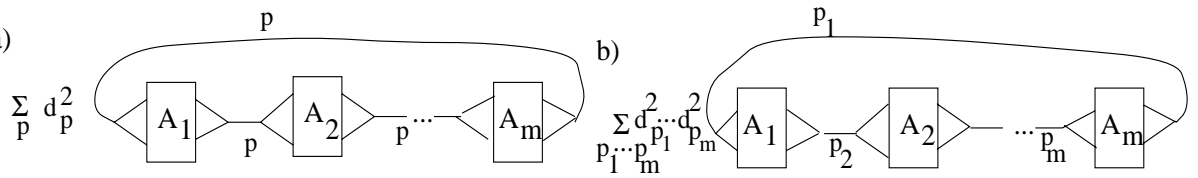

Figure 4.5.

$$
=\sum_{\mathrm{c}}^{\mathrm{B}_{1}} \frac{\mathrm{B}_{\mathrm{c}}^{2}}{\mathrm{\textrm {B } _ { 2 }}}=0
$$

Figure 4.6.

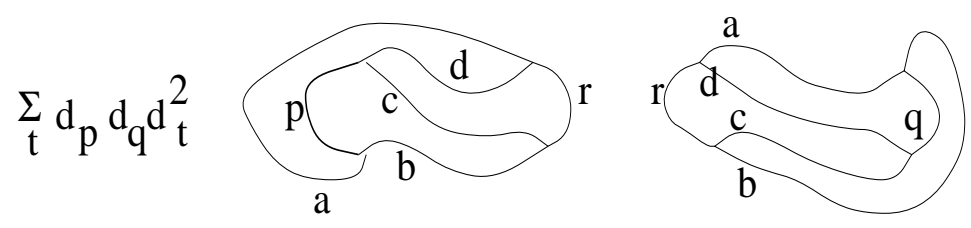

Figure 4.7.

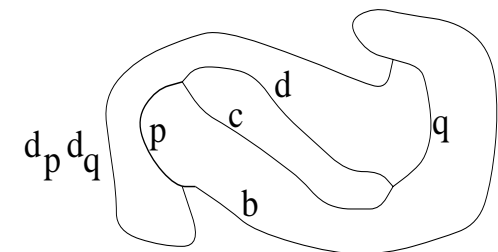

a

Figure 4.8 .

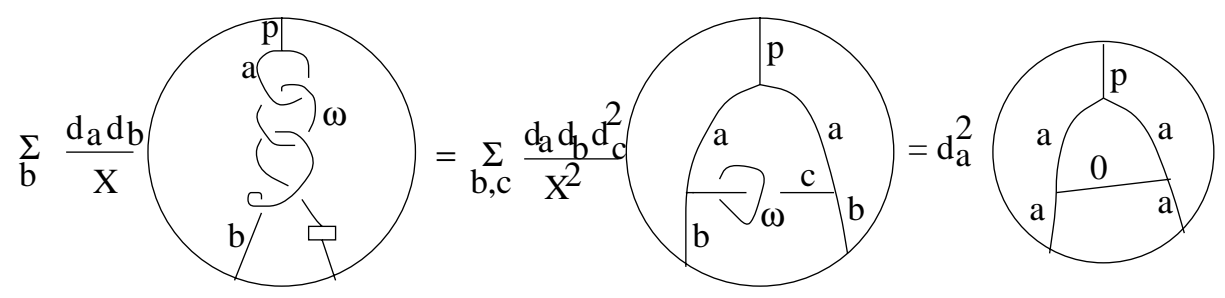

Figure 4.9 . 
As a consequence of this fact we get that our expression is equal to the one from Fig. 4.5. b), and then by applying the identity (2) from Fig. 3.6 several times we get the desired result.

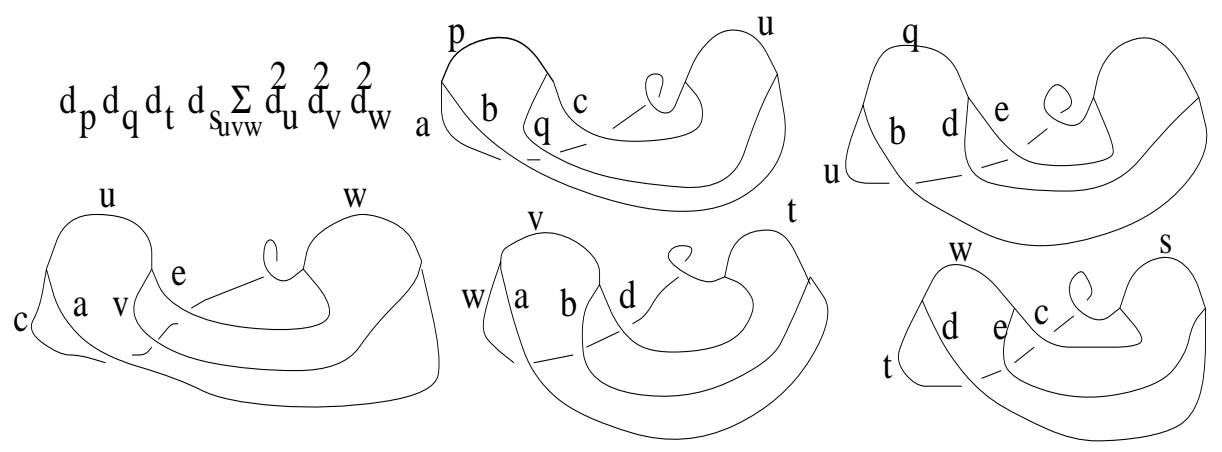

Figure 4.10 .

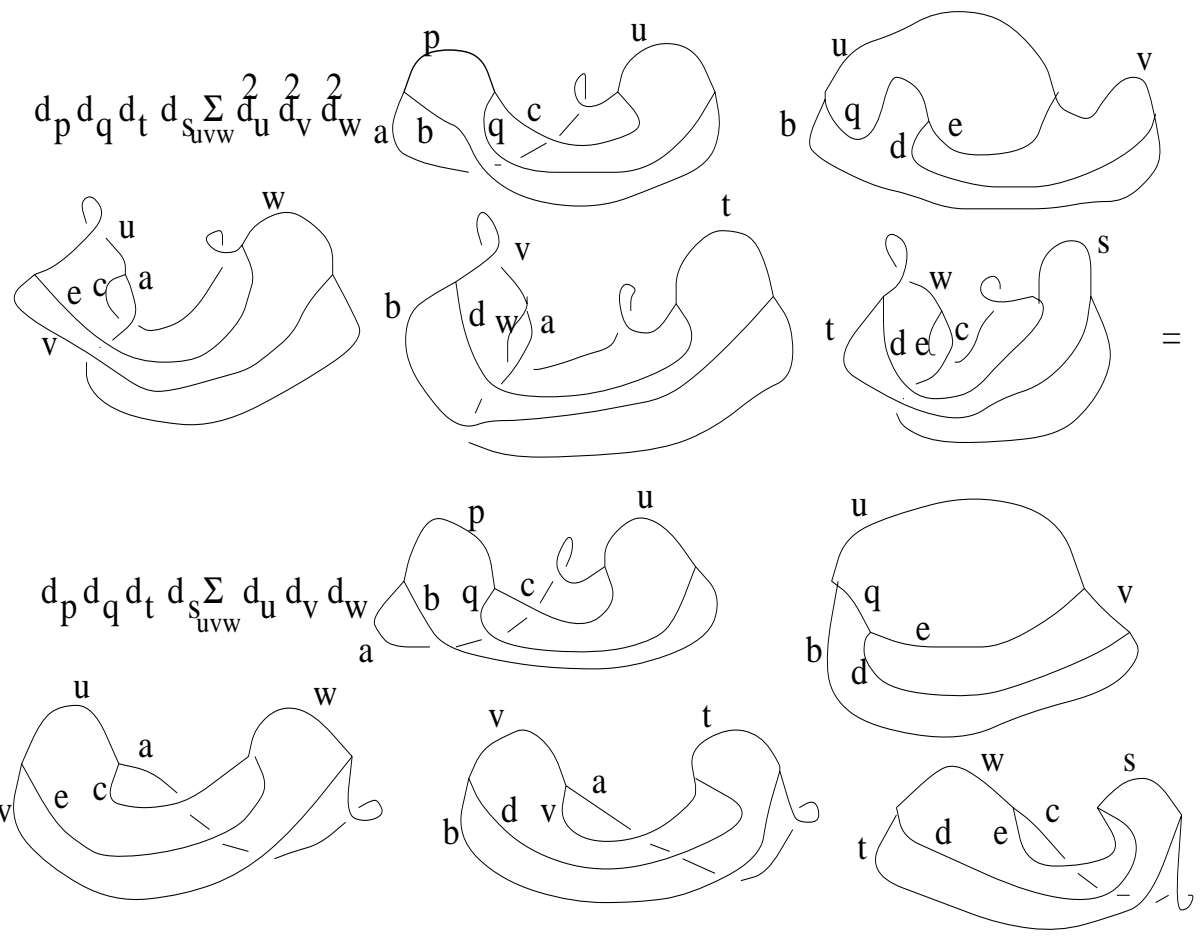

Figure 4.11. 
We can proceed with proving the compatibility conditions. The proofs are similar to the ones in $[\mathrm{FK}]$ and [G1], but one should note that they are simpler. First, the relations on a pair of pants are obviously satisfied. This can be seen at first glance for 1.a) and 1.c), then 1.d) is the third Reidemeister move, and 1.b) is equivalent to 1.c) (see $[\mathrm{FK}]$ or Chap. VI in $[\mathrm{T}]$ ).

For the proof of 2.a) we write $F P F \beta_{p a b} \otimes \beta_{p c d}=\sum_{q} c_{a b c d p q} \beta_{q a b} \otimes \beta_{q c d}$. Since we have a matrix multiplication here we see that the coefficient $c_{a b c d p q}$ is given by the diagram from Fig. 4.7.

By using Theorem 4.1 this becomes the expression from Fig. 4.8. Using identity (1) from Fig. 3.6. wee see that this is equal to $\delta_{p q}$ multiplied by the Greek letter $\theta$ diagram, therefore is equal to $\delta_{p q}$ and the identity is proved.

For 2.b) we have that $T_{3}^{-1} B_{23}^{-1} S^{2} \beta_{p a a}$ is equal to the first term in Fig. 4.9. We get the chain of equalities from this figure by pulling first the strand labeled by $\omega$ down and using the identity (2) from Fig. 3.6, and then using identity (3) from Fig. 3.6. The last term is equal to $\beta_{a a}$.

Now we describe the proof of the pentagon identity. We are interested in computing the coefficient of

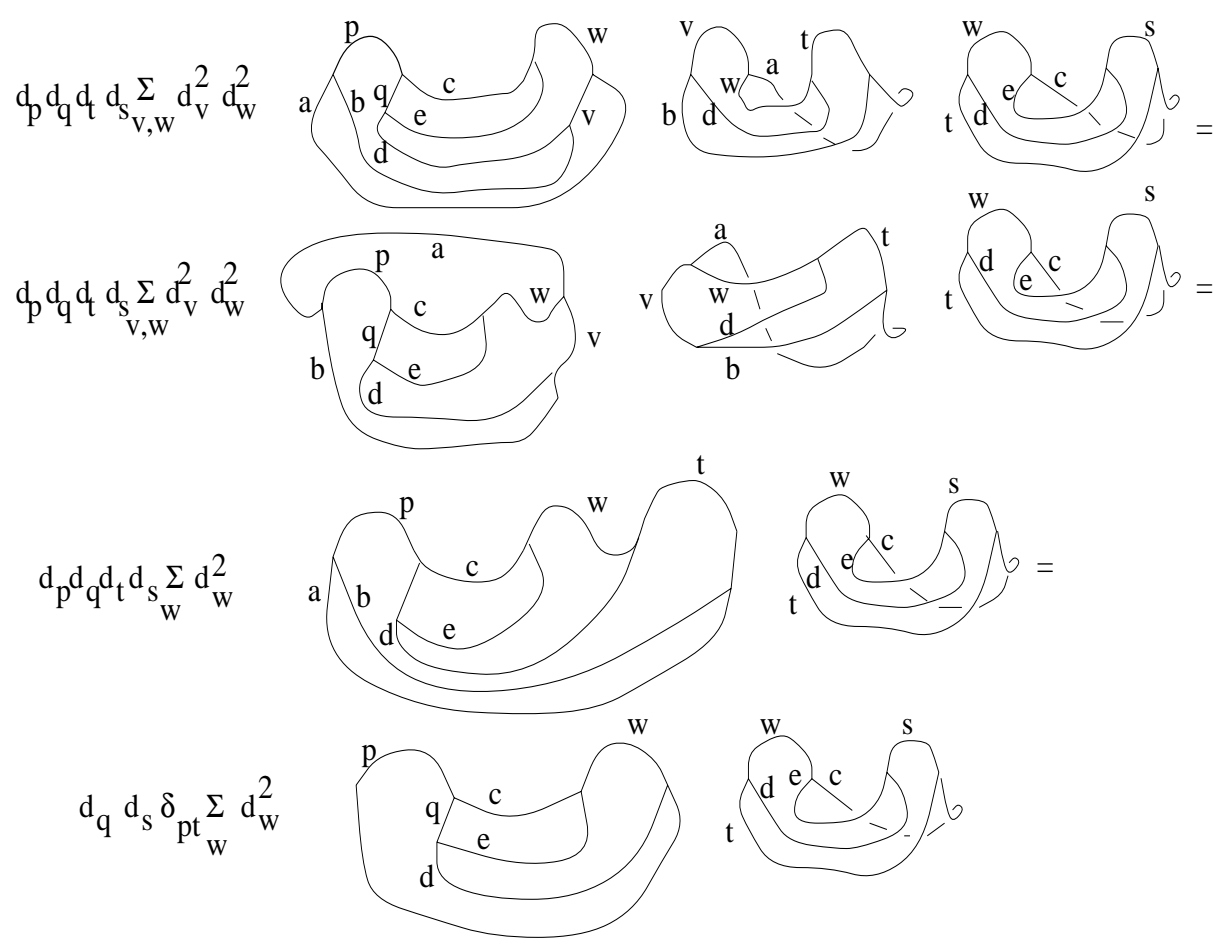

Figure 4.12 
$\beta_{\text {sde }} \otimes \beta_{\text {sct }} \otimes \beta_{\text {rab }}$ in $F^{(12)} R^{(2)} F^{(23)} R^{(2)} F^{(12)} R^{(2)} F^{(23)} R^{(2)} F^{(12)} \beta_{p a b} \otimes \beta_{p q c} \otimes \beta_{q d e}$. Again, by using the formula for matrix multiplication we get that this coefficient is described in Fig. 4.10.

By doing a flip in the third, fourth and fifth factor we get the first term from the equality shown in Fig. 4.11, which is further transformed into the second by applying three times 1.b). Apply Theorem 4.1 to contract with respect to $u$, then continue like in Fig. 4.12, namely pull the strand of $a$ over, then apply Theorem 4.1 for the sum over $v$ and then use for the last equality formula (1) in Fig. 3.6. Finally, if we use Theorem 4.1 once more and then formula (1) in Fig. 3.6, we get $\delta_{p t} \delta_{q s}$ times a diagram of the form of letter $\theta$. Hence the final answer is $\delta_{p t} \delta_{q s}$ and the identity is proved.

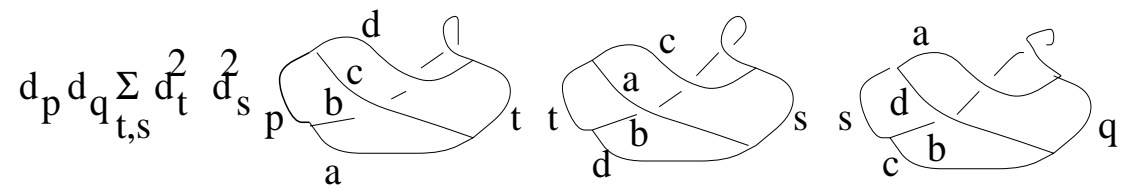

Figure 4.13

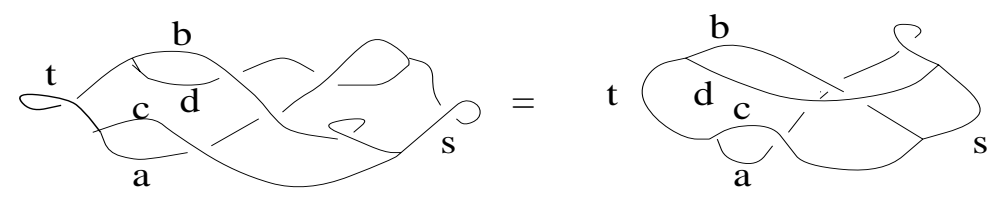

Figure 4.14.

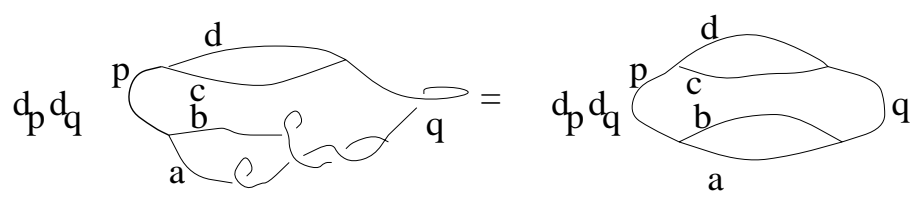

Figure 4.15

In order for the F-triangle to hold we have to show that the coefficient of $\beta_{q a b} \otimes \beta_{q c d}$ in $T_{3}^{(1)} F B_{23}^{(1)} F B_{23}^{(1)} F B_{23}^{(1)} \beta_{p a b} \beta_{p c d}$ is $\delta_{p q}$. The coefficient is given in Fig. 4.13 .

We transform the second factor as shown in Fig. 4.14 by first doing two flips and then using 1.b) twice. Then contract the product via Theorem 4.1 to get the first term from the equality from Fig. 4.15, then transform it into the second by using again 1.b). As before, this is equal to $\delta_{p q}$. 


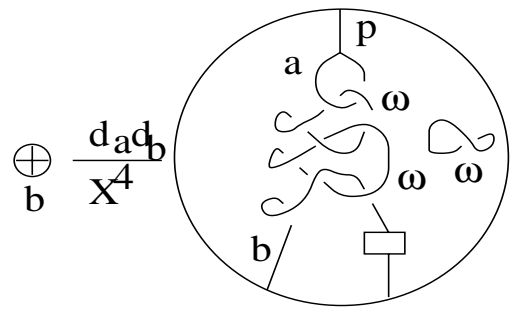

Figure 4.16 .

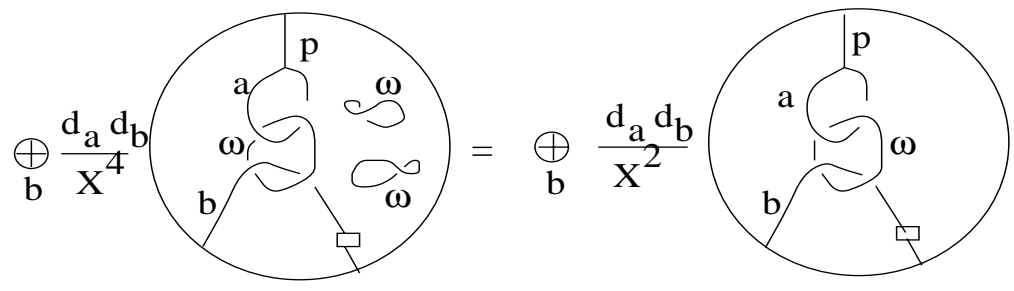

Figure 4.17

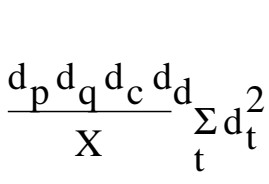

$\frac{d_{p} d_{q} d_{c} d_{d}}{X}{ }_{t} d_{t}^{2}$
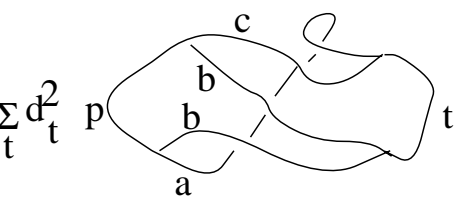

$\frac{\mathrm{d}_{\mathrm{p}} \mathrm{d}_{\mathrm{q}} \mathrm{d}_{\mathrm{c}} \mathrm{d}_{\mathrm{d}}}{X}$

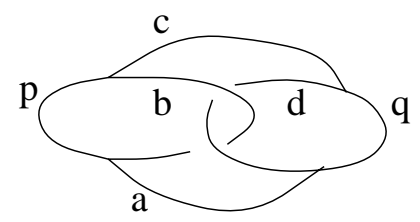

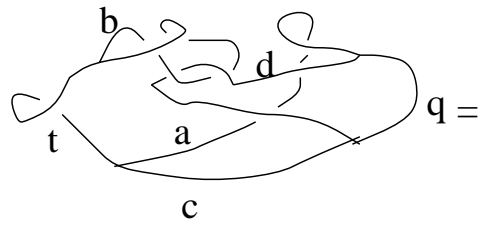

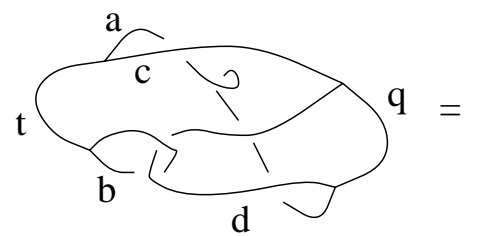

Figure 4.18.

In the case of the S-triangle, it is not hard to see that $C^{-1} B_{23}^{-1} T_{3}^{-2} S T_{3}^{-1} S T_{3}^{-1}$ $S \beta_{a a}$ is equal to the expression from Fig. 4.16. Lemma 3 in [L1] enables us to do Kirby moves over components labeled by $\omega$, so we get the first term from Fig. 4.17 , which is equal to the second one by Lemma 4 in [L1]. From here we continue like in the proof of 2.b). 
Let us prove 3.d). We have to show that the coefficient of $\beta_{q d c} \otimes \beta_{q d a}$ in $F S^{(2)} T_{3}^{(2)}\left(T_{1}^{(2)}\right)^{-1} B_{23}^{(2)} F \beta_{p a b} \otimes \beta_{p b c}$ is the same as the coefficient of this vector in $R^{(1)}\left(R^{(2)}\right)^{-1} F S^{(1)} F B_{23}^{(1)} B_{23}^{(2)} \beta_{p a b} \otimes \beta_{p b c}$. For the first one we have the sequence of equalities from Fig. 4.18, where the second equality is obtained by contracting via Theorem 4.1. For the second one we have the equalities from Fig. 4.19, where at the first step we used a combination of a flip and 1.b) and at the second step we contracted. By moving strands around the reader can convince himself that the two are equal.
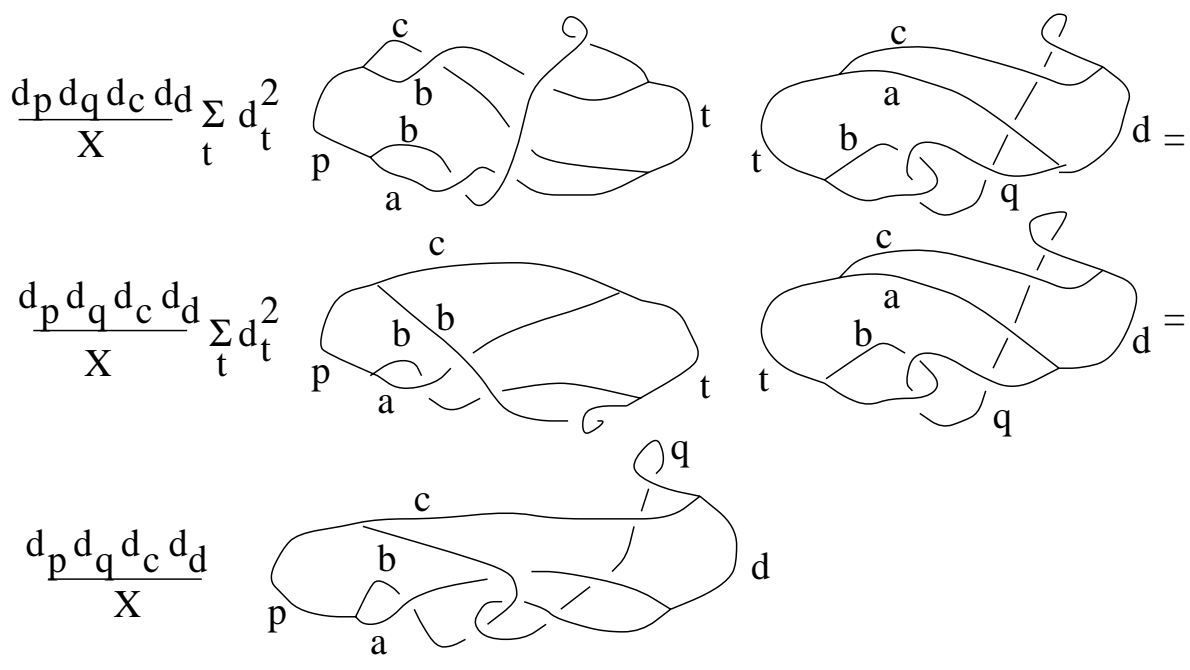

Figure 4.19 .

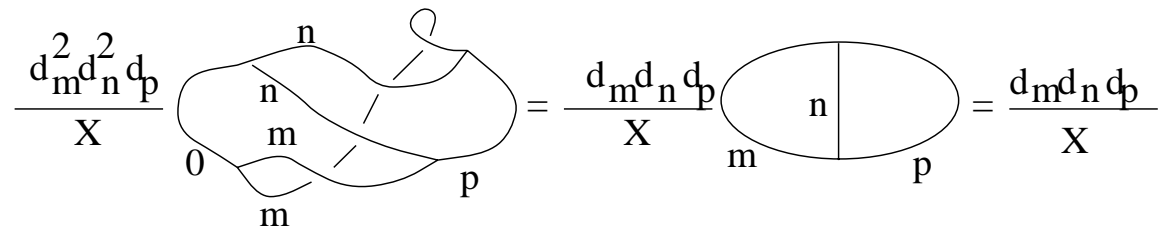

Figure 4.20.

The groups of relations 4, 5, and 6 are straightforward. Also, we see that the function $S$ has been chosen such that 7.a) holds. Let's prove 7.b). Here is the place where we see why we normalized the pairing the way we did. We have to prove that $d_{m}^{2} d_{n}^{2} X^{-2} F \beta_{0 m m} \otimes \beta_{0 n n}=\oplus_{p} d_{m} d_{n} d_{p} X^{-2} \beta_{p n m} \otimes \beta_{p m n}$. We see in Fig. 4.20 that this is true. 


\section{Some properties of invariants of 3-manifolds}

We begin this section with the generalization of Theorem 8 from [L1] (see also Proposition 10.1 in [BHMV1]) to surfaces with boundary.

Proposition 5.1. Let $\Sigma$ be a surface of genus $g$ with $n$ boundary components, and let $D$ be the DAP-decomposition of $\Sigma \times S^{1}$ whose decomposition circles are the components of $\partial \Sigma \times\{1\}$ and whose seams are of the form $\{x\} \times S^{1}$, with $x \in \partial \Sigma$. Then

$$
Z\left(\Sigma \times S^{1}, D, 0\right)=\sum_{j_{1}, j_{2}, \cdots, j_{n}} c_{j_{1}, j_{2}, \cdots, j_{n}} \beta_{j_{1} j_{1}} \otimes \beta_{j_{2} j_{2}} \otimes \cdots \otimes \beta_{j_{n} j_{n}}
$$

where $j_{1}, j_{2}, \cdots, j_{n}$ run over all labelings of $\partial \Sigma$ and $c_{j_{1}, j_{2}, \cdots, j_{n}}$ is the number of ways of labeling the diagram in Fig. 5.1 with integers $i_{k}$ such that at each node we have an admissible triple.

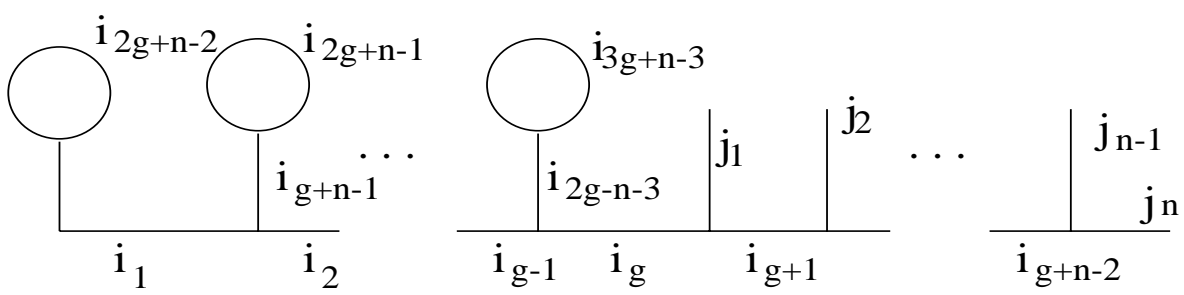

Figure 5.1.

Proof. Consider on $\Sigma$ a DAP-decomposition $D_{0}$ with decomposition curves as shown in Fig. 5.2. Put on $\Sigma \times I$ the DAP-decomposition $D^{\prime}$ that coincides with $D_{0}$ on $\Sigma \times\{1\}$, with $-D_{0}$ on $-\Sigma \times\{0\}$, and on $\partial \Sigma \times I$ there are no extra decomposition circles, and the seams are vertical (i.e. of the form $\{x\} \times I$ ).

It follows that $\left(\Sigma \times I, D^{\prime}, 0\right)$ is the mapping cylinder of $(i d, 0)$ (with vertical annuli no longer contracted like in the definition of the mapping cylinder from Section 2). The mapping cylinder axiom implies that

$$
Z\left(\Sigma \times I, D^{\prime}, 0\right)=\bigotimes_{j_{1}, j_{2}, \cdots, j_{n}} i d_{j_{1}, j_{2}, \cdots, j_{n}} \beta_{j_{1} j_{1}} \otimes \beta_{j_{2} j_{2}} \otimes \cdots \otimes \beta_{j_{n} j_{n}}
$$

where $i d_{j_{1}, j_{2}, \cdots, j_{n}}$ is the identity endomorphism on $V\left(\Sigma, D_{0},\left(j_{1}, j_{2}, \cdots, j_{n}\right)\right)$.

If we glue the ends of $\Sigma \times I$ via the identity map we get the e-3-manifold from the statement. The gluing axiom implies that in the formula above the identity matrices get replaced by their traces. Therefore

$$
Z\left(\Sigma \times S^{1}, D, 0\right)=\bigotimes_{j_{1}, j_{2}, \cdots, j_{n}} \operatorname{dim} V\left(\Sigma, D_{0},\left(j_{1}, j_{2}, \cdots, j_{n}\right)\right) \beta_{j_{1} j_{1}} \otimes \beta_{j_{2} j_{2}} \otimes \cdots \otimes \beta_{j_{n} j_{n}}
$$




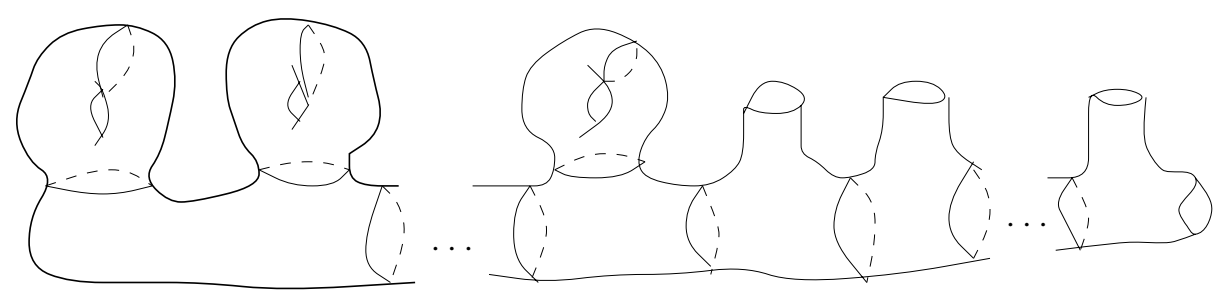

Figure 5.2.

On the other hand the gluing axiom for $V$ implies that $\operatorname{dim} V\left(\Sigma, D_{0},\left(j_{1}, j_{2}, \cdots, j_{n}\right)\right)=c_{j_{1}, j_{2}, \cdots, j_{n}}$, which proves the proposition.

The following result shows that the Kauffman bracket not only determines our TQFT, but also can be recovered from it. In the light of this theorem, a TQFT is the generalization to manifolds of the polynomial invariants of links. It is an analogue of Theorem 1.1 in [G2] which showed the presence of the skein relation of the Jones polynomial in the context of the Reshetikhin-Turaev TQFT. Before we state the theorem we have to introduce some notation.

Let us assume that the three e-manifolds $\left(M_{1}, D_{1}, 0\right),\left(M_{2}, D_{2}, 0\right)$ and $\left(M_{3}, D_{3}, 0\right)$ are obtained by gluing to the same e-manifold, via the same gluing map, the genus 2 e-handlebodies from Fig. 5.3 respectively, where the gluing occurs along the "exterior" punctured spheres. Note that the three handlebodies have the same structure on the "exterior" spheres, so they produce the same change of framing (if any) when gluing.
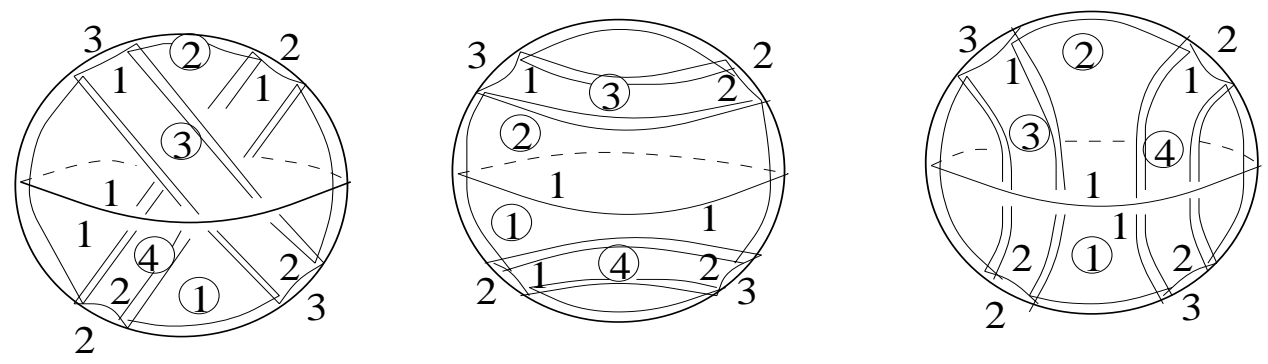

Figure 5.3.

The "interior" annuli of the handlebodies are part of the boundaries of our 3-manifolds. The gluing axiom implies that $V\left(\partial M_{i}, D_{i}\right)$ splits as a direct sum $V_{i} \bigoplus V_{i}^{\prime}$, where $V_{i}$ is the subspace corresponding to the labeling of the ends of the annuli by 1 . Moreover, the gluing axiom for $Z$ implies that $Z\left(M_{i}, D_{i}, 0\right)$ also splits as $v_{i} \oplus v_{i}^{\prime}$ where $v_{i} \in V_{i}$ and $v_{i}^{\prime} \in V_{i}^{\prime}$. On the other hand the spaces $V_{1}, V_{2}$ and $V_{3}$ are canonically isomorphic. Indeed, they have a common part, to which the vector spaces corresponding to the two annuli with ends labeled by 1 are attached 
via the map $x \rightarrow x \otimes \beta_{11} \otimes \beta_{11}$. Thus $v_{1}, v_{2}$ and $v_{3}$ can be thought as lying in the same vector space. With this convention in mind, the following result holds.

Theorem 5.1. The vectors $v_{1}, v_{2}$, and $v_{3}$ satisfy the Kauffman bracket skein relation

$$
v_{1}=A v_{2}+A^{-1} v_{3}
$$

Proof. By the gluing axiom for $Z$ we see that it suffices to prove the theorem in the case where $M_{1}, M_{2}$ and $M_{3}$ coincide with the three handlebodies (i.e. when the manifold to which they get glued is empty).

The first e-manifold is obtained by first taking the mapping cylinder of the homeomorphism on a pair of pants that takes the "right leg" over the "left leg" as shown in Fig. 5.4 (it should be distinguished from a move in the sense that it really maps one seam into the other), then composing it with the move $B_{23}^{(1)}$, and finally by expanding two annuli via moves of type $A^{-1}$.

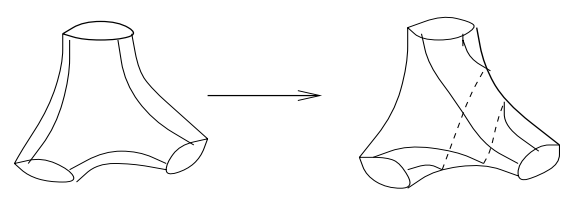

Figure 5.4.

We get

$$
v_{1}=B_{23} \hat{\beta}_{011} \otimes \beta_{011} \otimes \beta_{11} \otimes \beta_{11}+B_{23} \hat{\beta}_{211} \otimes \beta_{211} \otimes \beta_{11} \otimes \beta_{11}
$$

where for $x \in V_{a b c}$ we denote by $\hat{x}$ the vector in $\left(V_{a b c}\right)^{*}$ with the property that $<x, \hat{x}>=1$. By the definition of the pairing $\hat{\beta}_{011}=d_{1}^{2} X^{-2} \beta_{011}$ and $\hat{\beta}_{211}=$ $d_{1}^{2} d_{2} X^{-2} \beta_{211}$. The computation of $B_{23} \beta_{011}$ and $B_{23} \beta_{211}$ is described in Fig. 5.5.

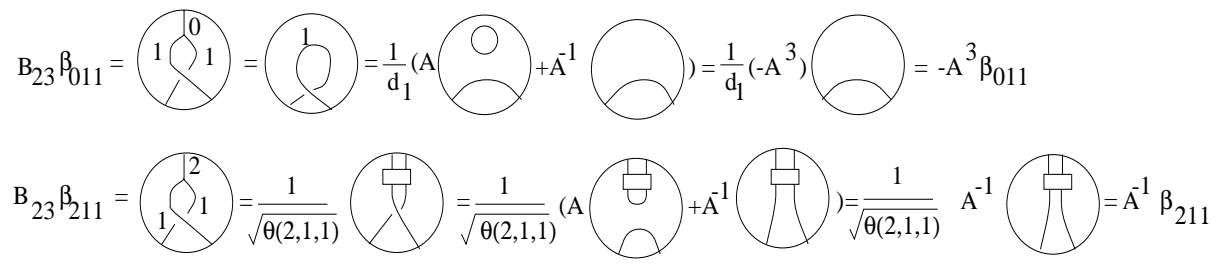

Figure 5.5.

Hence

$v_{1}=-A^{3} d_{1}^{2} X^{-2} \beta_{011} \otimes \beta_{011} \otimes \beta_{11} \otimes \beta_{11}+A^{-1} d_{1}^{2} d_{2} X^{-2} \beta_{211} \otimes \beta_{211} \otimes \beta_{11} \otimes \beta_{11}$. 
The second manifold can be obtained by gluing along a disk the mapping cylinders of two annuli. The mapping cylinder of an annulus has the invariant $\oplus_{a} \hat{\beta}_{a a} \otimes \beta_{a a}=\oplus_{a} d_{a}^{2} X^{-1} \beta_{a a} \otimes \beta_{a a}$, so after expanding a disk and gluing the two copies together we get $\oplus_{a, b} d_{a}^{2} d_{b}^{2} X^{-2} \beta_{0 a a} \otimes \beta_{0 b b} \otimes \beta_{a a} \otimes \beta_{b b}$. But we are only interested in the component of the invariant for which $a=b=1$, hence $v_{2}=$ $d_{1}^{4} X_{-2} \beta_{011} \otimes \beta_{011} \otimes \beta_{11} \otimes \beta_{11}$.

Finally, the third e-manifold is the mapping cylinder of the identity with two expanded annuli, hence

$$
v_{3}=-d_{1}^{2} X^{-2} \beta_{011} \otimes \beta_{011} \otimes \beta_{11} \otimes \beta_{11}+d_{1}^{2} d_{2} X^{-2} \beta_{211} \otimes \beta_{211} \otimes \beta_{11} \otimes \beta_{11} .
$$

The conclusion follows by noting that the diagram that gives the value of $d_{1}^{2}=\Delta_{1}$ is the unknot, hence $d_{1}^{2}=-A^{2}-A^{-2}$.

As a consequence of the theorem we will compute the formula for the invariant of the complement of a regular neighborhood of a link.

Proposition 5.2. Let $L$ be a framed link with $k$ components, and $M$ be the complement of a regular neighborhood of $L$. Consider on $\partial M$ the DAP-decomposition $D$ whose decomposition curves are the meridinal circles of $L$ (one for each component) and whose seams are parallel to the framing (see Fig. 5.6.a)). Then

$Z(M, D, 0)=\frac{1}{X} \sum_{n_{1} n_{2} \cdots n_{k}}<S_{n_{1}}(\alpha), S_{n_{2}}(\alpha), \cdots, S_{n_{k}}(\alpha)>_{L} \beta_{n_{1} n_{1}} \otimes \beta_{n_{2} n_{2}} \otimes \cdots \beta_{n_{k} n_{k}}$

where the sum is over all labels, and $\langle\cdot, \cdot, \cdots, \cdot\rangle_{L}$ is the link invariant defined in Section 3.

Proof. We assume that $L$ is given by a diagram in the plane with the blackboard framing. When $L$ is the unknot the invariant can be obtained from Proposition 5.1 applied to the case where $\Sigma$ is a disk, so in this situation $Z(M, D, 0)=$ $1 / X \Sigma_{n} d_{n}^{2} \beta_{n n}$ and the formula holds. By taking the connected sum of $k$ copies of the complement of the unknot, and using the gluing axiom for $Z$ we see that the formula also holds for the trivial link with $k$ components. Let us prove it in the general case. Put $Z(M, D, 0)=1 / X \sum_{n_{1} n_{2} \cdots n_{k}} c_{n_{1} n_{2} \cdots n_{k}} \beta_{n_{1} n_{1}} \otimes \beta_{n_{2} n_{2}} \otimes \cdots \beta_{n_{k} n_{k}}$. We want to prove that

$$
c_{n_{1} n_{2} \cdots n_{k}}=<S_{n_{1}}(\alpha), S_{n_{2}}(\alpha), \cdots, S_{n_{k}}(\alpha)>_{L} .
$$

Since by Theorem 5.1, $c_{11 \cdots 1}$ and $<S_{1}(\alpha), S_{1}(\alpha), \cdots, S_{1}(\alpha)>_{L}$ satisfy both the Kauffamn bracket skein relation, the equality holds when all indices are equal to 1 . If some of the indices are equal to 0 , the corresponding link components can be neglected (by erasing them in the case of the link, and by gluing inside solid tori in the trivial way in the case of the 3 -manifold). Therefore the equality holds if $n_{i}=0,1, i=1,2, \cdots, k$. 
a)

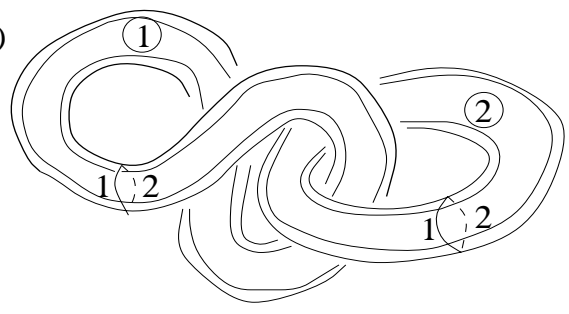

b)

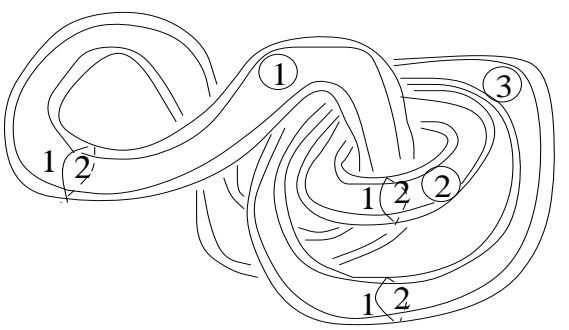

Figure 5.6.

For a tuple $\mathbf{n}=\left(n_{1}, n_{2}, \cdots, n_{k}\right)$ let $\mu(\mathbf{n})=\max \left\{n_{i} \mid i=1,2, \cdots, k\right\}$ and $\nu(\mathbf{n})=$ $\operatorname{card}\left\{i \mid n_{i}=\mu(\mathbf{n})\right\}$. We will prove (1) by induction on $(\mu(\mathbf{n}), \nu(\mathbf{n}))$, where the pairs are ordered lexicographically. Suppose that the property is true for all links and all tuples $\mathbf{n}^{\prime}$ with $\left(\mu\left(\mathbf{n}^{\prime}\right), \nu\left(\mathbf{n}^{\prime}\right)\right)<(\mu(\mathbf{n}), \nu(\mathbf{n}))$ and let us prove it for $(\mu(\mathbf{n}), \nu(\mathbf{n}))$.

Let $M_{0}$ be the product of a pair of pants with a circle. Put on $M_{0}$ a DAPdecomposition $D_{0}$ as described in Proposition 5.1. Then

$$
Z\left(M_{0}, D_{0}, 0\right)=\sum_{m n p} \delta_{m n p} \beta_{m m} \otimes \beta_{n n} \otimes \beta_{p p}
$$

where $\delta_{m n p}=1$ if $(m, n, p)$ is admissible and 0 otherwise.

Assume that in the tuple $\mathbf{n}=\left(n_{1}, n_{2}, \cdots, n_{k}\right), n_{k}=\mu(\mathbf{n})$. Glue $M_{0}$ to $M$ along the $k$-th torus of $M$ such that in the gluing process the DAP-decompositions of the two tori overlap. We get an e-manifold $\left(M_{1}, D_{1}, 0\right)$ that is nothing but the manifold associated to the link $L^{\prime}$ obtained from $L$ by doubling the last component (see Fig. 5.6. b)).

Let $Z\left(M_{1}, D_{1}, 0\right)=1 / X \Sigma d_{m_{1} m_{2} \cdots m_{k}, m_{k+1}} \beta_{m_{1} m_{1}} \otimes \beta_{m_{2} m_{2}} \otimes \cdots \beta_{m_{k+1} m_{k+1}}$. The gluing axiom, together with relation 6.a) from Section 3 imply that $d_{m_{1}, m_{2}, \cdots, m_{k+1}}$ $=\sum_{p} \delta_{m_{k} m_{k+1} p} c_{m_{1}, m_{2}, \cdots, m_{k-1}, p}$. In particular

$$
d_{n_{1}, n_{2}, \cdots, n_{k-1}, n_{k}-1,1}=c_{n_{1}, n_{2}, \cdots, n_{k}-2}+c_{n_{1}, n_{2}, \cdots, n_{k}} .
$$

Applying the induction hypothesis we get

$$
\begin{aligned}
c_{n_{1} n_{2} \cdots n_{k}} & =<S_{n_{1}}(\alpha), \cdots, S_{n_{k-1}}(\alpha), S_{n_{k}-1}(\alpha), \alpha>_{L^{\prime}} \\
- & <S_{n_{1}}(\alpha), \cdots, S_{n_{k-1}}(\alpha), S_{n_{k}-2}(\alpha)>_{L} .
\end{aligned}
$$

But $<S_{n_{1}}(\alpha), \cdots, S_{n_{k-1}}(\alpha), S_{n_{k}-1}(\alpha), \alpha>_{L^{\prime}}=<S_{n_{1}}(\alpha), \cdots, S_{n_{k-1}}(\alpha)$, $\alpha S_{n_{k}-1}(\alpha)>_{L}$ and since $S_{n_{k}}(\alpha)=\alpha S_{n_{k}-1}(\alpha)-S_{n_{k}-2}(\alpha)$ (see [L1]), we obtain the equality in (1) and the proposition is proved.

Corollary. If $M$ is a closed 3-manifold obtained by performing surgery on the framed link $L$ with $k$ components, then

$$
Z(M, 0)=X^{-k-1} C^{-\sigma}<\omega, \omega, \cdots, \omega>_{L}
$$


where $\Sigma$ is the signature of the linking matrix of $L$.

Proof. We may assume that $L$ is given by a link diagram in the plane and its framing is the blackboard framing. Let $\left(M_{1}, D_{1}, 0\right)$ be the e-3-manifold associated to $L$ as in the statement of Proposition 5.2. Consider the e-manifold $\left(M_{2}, D_{2}, 0\right)$ where $M_{2}$ is the solid torus and $D_{2}$ is described in Fig. 5.7. Applying Proposition 5.2 to the unknot we see that the invariant of this e-manifold is $1 / X \Sigma_{n} d_{n}^{2} \beta_{n n}$.

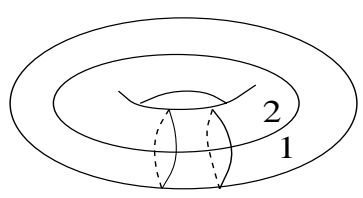

Figure 5.7.

If we glue $k$ copies of this manifold to $M_{1}$ such that the DAP-decompositions overlap we get $M$. In the gluing process the framing changes by $-\sigma\left(L_{1}, L_{2}, L_{3}\right)$ (see Section 1) where $L_{1}$ is the kernel of $H_{1}\left(\partial M_{1}\right) \rightarrow H_{1}\left(M_{1}\right), L_{2}$ is the Lagrangian space spanned in $H_{1}(\partial M)$ by the meridinal circles of the link, and $L_{3}$ is the one spanned by the curves that give the framing. It is a standard result in knot theory that $-\sigma\left(L_{1}, L_{2}, L_{3}\right)=\sigma$, the linking matrix of $L$. Using the gluing axiom for $Z$ we get

$$
\begin{aligned}
Z(M, \sigma) & =X^{-k-1} \sum_{n_{1}, n_{2}, \cdots, n_{k}} d_{n_{1}}^{2} d_{n_{2}}^{2} \cdots d_{n_{k}}^{2}<S_{n_{1}}(\alpha), S_{n_{2}}(\alpha), \cdots, S_{n_{k}}(\alpha)>_{L} \\
& =X^{-k-1}<\omega, \omega, \cdots, \omega>_{L}
\end{aligned}
$$

hence

$$
Z(M, 0)=X^{-k-1} C^{-\sigma}<\omega, \omega, \cdots, \omega>_{L}
$$

We make the final remark that this gives the invariants of 3-manifolds normalized as in [L1]. A similar argument can be used to prove the invariant formula for three manifolds with boundary [G3].

\section{References}

[A] M. F. Atiyah, The Geometry and Physics of knots, Lezioni Lincee, Accademia Nationale de Lincei, Cambridge Univ. Press, 1990.

[BHMV1] C. Blanchet, N. Habegger, G. Masbaum, P. Vogel, Topological quantum field theories derived from the Kauffman bracket, Topology 31(1992), 685-699.

[BHMV2] C. Blanchet, N. Habegger, G. Masbaum, P. Vogel, Topological quantum field theories derived from the Kauffman bracket, Topology bf 31 (1995), 883-927. 
[Ce] J. Cerf, La stratification naturelle et le théoreme de la pseudo-isotopie, Publ. Math. I.H.E.S. 39 (1969), 5-173.

[FK] Ch. Frohman, J. Kania-Bartoszynska, $S O(3)$ topological quantum field theory, Comm. Anal. Geom. 4 (1996), no. 4, 589-679.

[G1] R. Gelca, SL(2,C) topological quantum field theory with corners, preprint, 1995.

[G2] R. Gelca, The quantum invariant of the complement of a link, preprint, 1996.

[G3] R. Gelca, On the formula of the quantum invariant for three manifolds with boundary, preprint, 1996.

[HT] A. Hatcher, W. Thurston, A presentation of the mapping class group of a closed orientable surface, Topology 19 (1980), 221-237.

$[\mathrm{J}]$ V. F. R. Jones, Polynomial invariants of knots via von Neumann algebras, Bull. Amer. Math. Soc. 12 (1985), 103-111.

[K1] L. Kauffman, States models and the Jones polynomial, Topology 26 (1987), 395-407.

[K2] L. Kauffman, Knots and Physics, World Scientific, 1991.

[Ki] R. Kirby, A calculus for framed links in $S^{3}$, Inventiones Math. 45 (1987), 35-56.

[KM] R. Kirby, P. Melvin, The 3-manifold invariants of Witten and Reshetikhin-Turaev for sl(2, C), Inventiones Math. 105 (1991), 547-597.

[Ko] T. Kohno, Topological invariants for 3-manifolds using representations of the mapping class groups, Topology 31 (1992), 203-230.

[L1] W. B. R. Lickorish, The skein method for three-manifold invariants, J. Knot Theor. Ramif. 2 no. 2 (1993), 171-194.

[L2] W. B. R. Lickorish, Skeins and handlebodies, Pac. J. Math. 159 No. 2 (1993), 337-350.

[MS] G. Moore, N. Seiberg, Classical and quantum field theory, Commun. Math. Phys. 123 (1989), 177-254.

[RT] N. Yu. Reshetikhin, V. G. Turaev, Invariants of 3-manifolds via link polynomials and quantum groups, Inventiones Math. 103 (1991), 547-597.

[R] J. Roberts, Skeins and mapping class groups, Math. Proc. Camb. Phil. Soc. 115 (1995), 53-77.

[T] V. G. Turaev, Quantum invariants of Knots and 3-manifolds, de Gruyter Studies in Mathematics, de Gruyter, Berlin-New York 1994.

[Wa] K. Walker, On Witten's 3-manifold invariants, preprint, 1991.

[W] C. T. C. Wall, Non-additivity of the signature, Inventiones Math. 7 (1969), 269-274.

[We] H. Wenzl, On sequences of projections, C. R. Math. Rep. Acad. Sci. IX (1987), 5-9.

[Wi] E. Witten, Quantum field theory and the Jones polynomial, Comm. Math. Phys. 121 (1989), 351-399.

Răzvan Gelca

Department of Mathematics

The University of Iowa

Iowa City, IA 52242

USA

e-mail: rgelca@math.uiowa.edu

and

Institute of Mathematics of Romanian Academy

P.O.Box 1-764

70700 Bucharest, Romania

(Received: March 4, 1996) 